\title{
DESARROLLO DE UN ENTORNO VIRTUAL PARA LA SIMULACIÓN DE MANIOBRAS ELÉCTRICAS EN SUBESTACIONES: UN CASO PRÁCTICO
}

\author{
David Antonio Riveros-Hernández ${ }^{1}$, Diego Hernando Nausan-García ${ }^{2}$, Diana \\ Stella García-Miranda ${ }^{3}$, José Ignacio Palacios-Osma ${ }^{4}$ \\ ${ }^{1}$ Estudiante de Ingeniería Eléctrica, proyecto curricular de Ingeniería Eléctrica, Facultad de Ingeniería, Universidad \\ Distrital Francisco José de Caldas, Bogotá, Colombia \\ ${ }^{2}$ Estudiante de Ingeniería Eléctrica, proyecto curricular de Ingeniería Eléctrica, Facultad de Ingeniería, Universidad \\ Distrital Francisco José de Caldas, Bogotá, Colombia \\ Correo electrónico:dhnausang@correo.udistrital.edu.co \\ ${ }^{3}$ Magíster en Ingeniería Eléctrica, profesora del proyecto curricular de Ingeniería Eléctrica, Facultad de Ingeniería, \\ Universidad Distrital Francisco José de Caldas, Bogotá, Colombia \\ ${ }^{4}$ Magíster en e-learning y redes sociales, coordinador y profesor del proyecto curricular, Maestría en \\ Telecomunicaciones Móviles, Facultad de Ingeniería, Universidad Distrital Francisco José de Caldas, Bogotá, Colombia
}

Fecha de recibido: 10 de octubre del $2016 \quad$ Fecha de aprobado: 5 de marzo del 2017

Cómo citar este artículo: D. A. Riveros-Hernández, D. H. Nausan-García, D. S. Miranda, J. I. Palacios-Osma, "Desarrollo de un entorno virtual para la simulación de maniobras eléctricas en subestaciones: un caso práctico", Ingeniería Solidaria, vol. 13, n. ${ }^{2} 22$, mayo de 2017, pp. 55-84. doi: http://dx. doi.org/10.16925/in.v13i22.1752

Resumen. Introducción: resultado de una investigación que pretende mostrar la importancia de la inclusión del simulador de subestaciones eléctricas en la asignatura que lleva el mismo nombre (Facultad de Ingeniería, Universidad Distrital Francisco José de Caldas). Se propone una versión mejorada del simulador que incluya todas las configuraciones existentes y los módulos de diseño. Metodología: se emplearon encuestas muestrales para conocer la percepción de los estudiantes frente al simulador de subestaciones eléctricas, como metodología de aprendizaje y su incidencia en las cifras de repitencia. Se realiza una síntesis de la forma en que se desarrolló el software y su validación mediante una simulación que permite mostrar las ventajas de su uso para el tema de maniobras eléctricas, junto con las modificaciones recientes. Resultados: se muestra la potencialidad de la herramienta mediante la simulación de una maniobra eléctrica. A través de las encuestas muestrales, se obtuvo que más de un $80 \%$ de los estudiantes encuestados afirman que el simulador fue una herramienta útil en el tema de maniobras eléctricas, ya que presentaba mayor dificultad de aprendizaje entre los estudiantes; por ello, puede contribuir a reducir los índices de repitencia. Conclusiones: la inclusión del simulador permitió demostrar la utilidad de esta herramienta virtual para la comprensión de procesos de operación y mantenimiento de instalaciones de alta y extra alta tensión (subestaciones eléctricas), enmarcado en el concepto de laboratorios virtuales, válido para la formación de estudiantes de ciencias e ingenierías, de manera que contribuya a mejorar su desempeño en diferentes asignaturas.

Palabras clave: índice de repitencia, laboratorios virtuales, simuladores, subestaciones eléctricas. 


\title{
DeVElopment of A VirTual ENVIRONMENT For the Simulation of Electrical Maneuvers in Substations: A Case Study
}

\begin{abstract}
Introduction: This is the result of a research project that intends to show the importance of including the electrical substation simulator in the course that bears the same name (School of Engineering, Universidad Distrital Francisco José de Caldas). We propose an improved version of the simulator that includes all existing configurations and design modules. Method: Sample surveys were used to find out the students' perception of the electric substation simulator as a learning methodology and its effect on course repetition figures. A summary of how the software was developed and its validation through a simulation that allows to show the advantages of its use for electrical maneuvers, along with recent modifications, is provided. Results: The potentiality of the tool is shown by the simulation of an electric maneuver. Through the sample surveys, it was obtained that more than $80 \%$ of the students affirm that the simulator was a useful tool for electrical maneuvers, since this topic was very difficult to learn; therefore, it can contribute to reduce course repetition rates. Conclusions: The inclusion of the simulator demonstrated the usefulness of this virtual tool for understanding the operation and maintenance of high and extra high voltage installations (electrical substations). This is framed by the concept of virtual laboratories and is valid for the training of science and engineering students, thus contributing to improve their performance in different subjects.
\end{abstract}

Keywords: course repetition rate, virtual laboratory, simulator, electrical substations.

\section{DESENVOLVIMENTO DE UM AMBIENTE VIRTUAL PARA A SIMULAÇÃo DE MANOBRAS ELÉTRICAS EM SUBESTAÇÕES: UM CASO PRÁTICO}

Resumo. Introdução: este artigo é resultado de uma pesquisa que pretende mostrar a importância da inclusão do simulador de subestações elétricas na disciplina com o mesmo nome, da Faculdade de Engenharia da Universidad Distrital Francisco José de Caldas. Propõe-se uma versão melhorada do simulador que inclua todas as configurações existentes e os módulos de desenho. Metodologia: foram empregados questionários amostrais para conhecer a percepção dos estudantes ante o simulador de subestações elétricas, como metodologia de aprendizagem e sua incidência nas cifras de reprovação. Realiza-se uma síntese da forma em que o software foi desenvolvido e sua validação mediante uma simulação que permite mostrar as vantagens de seu uso para o tema de manobras elétricas, junto com as modificações recentes. Resultados: mostra-se a potencialidade da ferramenta mediante a simulação de uma manobra elétrica. Por meio de questionários amostrais, obteve-se que mais de $80 \%$ dos estudantes questionados afirmam que o simulador foi uma ferramenta útil no tema de manobras elétricas, já que apresentava maior dificuldade de aprendizagem entre os estudantes; por isso, pode contribuir para reduzir os índices de reprovação. Conclusões: a inclusão do simulador permitiu demonstrar a utilidade dessa ferramenta virtual para a compreensão de processos de operação e manutenção de instalações de alta e extra-alta tensão (subestações elétricas), delimitada no conceito de laboratórios virtuais, válido para a formação de estudantes de ciências e engenharias, de maneira que contribua para melhorar seu desempenho em diferentes disciplinas.

Palavras-chave: índice de reprovação, laboratório virtual, simulador, subestações elétricas. 


\section{Introducción}

El empleo de la tecnología ha facilitado la capacidad de comprender complejos aspectos técnicos [1] en la enseñanza de ciencias e ingenierías, mediante el desarrollo de herramientas didácticas que soporten el proceso de enseñanza-aprendizaje en el entorno educativo a través de escenarios interactivos e innovadores [2]. Uno de esos escenarios son los laboratorios virtuales. Según el concepto de la UnESCO, estos se definen como "un espacio electrónico de trabajo concebido para la colaboración y la experimentación a distancia con objeto de investigar o realizar otras actividades creativas, $y$ elaborar y difundir resultados mediante tecnologías difundidas de información y comunicación" [3, p. 918]. Otra definición más precisa considera que un laboratorio virtual, "es (son) instrumento(s) simulado(s) contenido(s) en uno o más ordenadores, conectados o no entre sí, con capacidad de gestión y/o aprendizaje de contenido" [4, p. 65]. La necesidad del hombre de comprender, interactuar y vivenciar los procesos y maniobras implicados en un sistema y en un ambiente controlado, dan origen al simulador [5]. Un simulador es un sistema que permite reproducir comportamientos de otros sistemas de manera semejante a como sucedería en el mundo real [5]; por extensión, se concluye que un laboratorio virtual es un simulador.

Resulta útil, en el ámbito académico, el desarrollo de herramientas virtuales como una tendencia pedagógica para que el estudiante adquiera las competencias y pueda participar activamente mediante la experimentación, el análisis y la toma de decisiones [3]. Asimismo, diversos sectores, tales como la medicina, el sector automotriz, aeronáutico y eléctrico, han desarrollado con éxito aplicaciones que imitan características físicas de objetos reales [1]. Por ejemplo, en medicina se ha implementado un modelo del cuerpo humano virtual, con el fin de probar la eficiencia de procedimientos quirúrgicos o de fármacos sin necesidad de utilizar personas que padecen la patología a curar. En la milicia, se han creado diferentes simuladores capaces de recrear diferentes entornos peligrosos, o con un alto grado de estrés, y simularlos las veces que sean necesarios, hasta que el soldado adquiera la habilidad de tomar las decisiones más acertadas en dicho entorno.

\subsection{Laboratorios virtuales en el proceso de enseñanza-aprendizaje}

Por consiguiente, es posible justificar la idea de desarrollar entornos virtuales en ciencias en las que la experimentación sea un factor clave de aprendizaje. De esta manera, ingenierías y áreas de ciencias físicas requieren para su aprendizaje conocimientos teóricos y elementos técnico-científicos que, enlazados a métodos empíricos, permitan fortalecer el proceso de construcción de aprendizaje. Estos campos de estudio e investigación deben estar apoyados de herramientas que permitan a los estudiantes consolidar los conocimientos teóricos adquiridos, mediante la práctica de los mismos en entornos reales interactivos.

Una de las principales dificultades es la realización de prácticas que permitan afianzar los conocimientos adquiridos, debido a que no se cuenta con la infraestructura necesaria, es decir, espacios físicos reales, equipos e instrumentación para ir de un aula de clase a un espacio de trabajo profesional. Por tal motivo, surgen los laboratorios virtuales como una forma de facilitar la realización de prácticas o experiencias a un mayor número de estudiantes, aunque alumnos y laboratorios no coincidan en un mismo espacio físico [6].

Como ventajas de la metodología virtual, con el uso de laboratorios virtuales que permiten justificar la implementación de estos en instituciones de educación superior aplicado a diferentes áreas del conocimiento, se pueden mencionar las siguientes:

- Un estudiante puede experimentar sin miedo a sufrir o provocar accidentes, realizando cuantas veces sea necesaria la misma práctica hasta obtener las competencias necesarias, mejorando las habilidades de resolución de problemas $[6,7]$.

- El estudiante puede asistir al laboratorio en cualquier momento, con la posibilidad de seleccionar las temáticas de acuerdo con sus necesidades de aprendizaje, además de recibir la asesoría de sus profesores para facilitar la comprensión de los temas asignados al laboratorio a realizar [8].

- El uso de Internet permite la experimentación asíncrona, ampliando la oferta de horarios para el estudiante en formación, de modo que sea un proceso de interacción mucho más fácil $[6,9]$.

- Permite al estudiante conocer elementos físicos de un laboratorio real o una instalación física cuando el acceso a ellos sea limitado. 
No obstante, no se puede sustituir del todo la experiencia práctica altamente enriquecedora en un entorno real, pues existen prácticas que solo pueden realizarse en campo. Un laboratorio virtual puede ser una herramienta complementaria valiosa en experiencias educativas tales como: teoría de circuitos, sistemas de control y dinámica de fluidos [6].

La diversidad de plataformas virtuales generadas, tanto por universidades, como por empresas, se ha dado con el objetivo de mejorar las competencias en diferentes áreas del conocimiento. En tal sentido, los laboratorios virtuales fortalecen la formación académica al contribuir a la comprensión de los diferentes procesos físicos. Lo anterior motiva a que muchas universidades trabajen en el desarrollo de nuevos ambientes virtuales aplicados a la enseñanza y la investigación, con el propósito de incentivar el uso de los mismos para la formación integral [3].

Es así que se avanza con mayor rapidez a una sociedad del conocimiento basada en el uso de las tecnologías de la información y las comunicaciones (TIC), en el que cobra sentido un concepto denominado "sociedad del aprendizaje" [10]. En este tipo de sociedad, se aplican los procesos de gestión del conocimiento en todos sus niveles y a través de todos los dispositivos tecnológicos que disponen las TIC [10]. Con plataformas de ambientes educativos virtuales se logra crear un contexto de simulación que influye en un proceso de aprendizaje en el que todos los estudiantes puedan ser partícipes e interactuar en la relación profesor-alumno, de manera que esto converja en una mejor comunicación que facilite el proceso de enseñanza-aprendizaje. A partir del uso de las Tic diferentes instituciones de educación superior, e incluso la industria, han logrado crear mundos virtuales capaces de facilitar y optimizar procesos que, en un pasado, implicaban mayores costos y tiempo [11].

El fortalecimiento de estas herramientas virtuales ahonda en un proceso de construcción de conocimiento, tanto de manera individual, como de manera colectiva. Bajo este contexto, el laboratorio virtual permite obtener resultados y experiencias que se pueden contrastar con la realidad. Diversos estudios evidencian cómo los estudiantes desarrollan nuevos conceptos y conocimiento en los laboratorios virtuales mediante el uso de estrategias constructivistas al proceso de enseñanza e investigación, a través de la estructuración de tareas complejas y automatización de rutinas [12]. Así, las instituciones de educación podrían adoptar los llamados "ambientes virtuales educativos", a fin de cumplir con el "aprendizaje por experiencia y colaboración” $[13,14]$.

\subsection{Desarrollos virtuales en ciencias e ingenierías, un estado del arte}

Siemens, National Instruments y otras industrias que se enfocan en el desarrollo de aplicaciones virtuales, han puesto en marcha simuladores para propósitos educativos y de ingeniería tales como Tecnomatix Jack, Tecnomatix Plant Simulation, Matlab, y Comsol Multiphysics, entre otros, que han impulsado no solo la visualización de modelos, sino también la realización de diferentes procesos de manufactura que convergen en una reducción de costos y de tiempo [11, 15].

Así, entonces, para el área de ciencias físicas, uno de los desarrollos más destacados es el proyecto Virtual Community Collaborating Space for Science Education (vCCSSE), el cual surge como una colaboración entre varias instituciones del Reino Unido, España, Polonia, Grecia y Finlandia, entre el 2006 y el 2009, y cuya coordinación está bajo la Universidad Valahia de Targoviste, en Rumania. El objetivo principal del vccsse fue adaptar, desarrollar, probar, implementar y difundir módulos de formación, metodologías de enseñanza y estrategias pedagógicas basadas en el uso de la instrumentación virtual en diferentes áreas de la ciencia (física, química, biología). El proyecto se demostró con experimentos simples como las leyes de gases (Charles, Gay-Lussac, Boyle-Marriote), transporte de calor (termodinámica) y circuitos eléctricos [16]. Otro de los proyectos destacados es TEALSim, desarrollado por el equipo Technology Enabled Active Learning (TEAL) del Massachusetts Institute of Technology (MIT), cuyo objetivo es incrementar el entendimiento conceptual y analítico de los estudiantes en fenómenos electromagnéticos [16].

En las áreas de procesos tecnológicos se destacan Ironmaking, desarrollado por la Universidad RWTH Aachen, en Alemania, hacia el 2008, que se caracteriza por realizar procesos físicos, químicos, hidráulicos y mecánicos con sus asociadas reacciones y está enfocado en la producción y uso del hierro. La plataforma virtual de aprendizaje CVD, desarrollada por el Departamento de Ingeniería Química de la Universidad Estatal de Oregón, en 
Estados Unidos, tiene como objetivo la simulación de procesos químicos (transferencia de masa y reacciones químicas) [16].

En el campo de la ingeniería no robótica, se destacan laboratorios virtuales para sistemas de control aplicados a plantas, como, por ejemplo, los sistemas de almacenamiento de líquidos basados en modelos matemáticos de ecuaciones diferenciales ordinarias, diseñados por la Universidad Eslovaca de Tecnología en Bratislavia (Eslovaquia). El concepto de laboratorio virtual multiplataforma para propósitos de educación, desarrollado por la Universidad Politécnica de Catalunya (España), demuestra los avances en creación de entornos para experimentos tales como levitación magnética y diseño de sistemas de control (PID y control óptimo). Cabe destacar a TriLab como un entorno virtual desarrollado por la Universidad Loughborough en el Reino Unido, el cual combina tres modos de acceso (práctico, virtual y remoto) en un solo paquete de software mediante LABVIEW, yes utilizado para demostrar los conceptos de sistemas de control por medio de la simulación de modelos relacionados con procesos físicos. De la misma forma, investigadores de la Universidad de Firat, en Turquía, desarrollaron un laboratorio virtual de máquinas eléctricas usando Hypertext Markup Language (HTML), Active Server Pages (ASP) y C++. El objetivo del mencionado laboratorio es mejorar la experimentación con máquinas eléctricas, de modo que los estudiantes sean capaces de observar los efectos de carga de un motor síncrono o cambiar parámetros del mismo tales como frecuencia, voltaje de entrada o tiempos de simulación. El instituto de tecnología Stevens, en Estados Unidos, desarrolló un laboratorio virtual online para la enseñanza de la dinámica de máquinas en estudiantes de ingeniería mecánica, mediante la interacción en un ambiente virtual a través de avatares creados por el usuario, en los que las leyes físicas son aplicadas [16].

Para el campo de ingeniería robótica, se destaca Roboualab como un laboratorio virtual y remoto desarrollado en el 2007 por la Universidad de Alicante (España), como evolución de Robolab, iniciado en 1999. Permite a los estudiantes practicar comandos de posicionamiento de un robot industrial y está basado en Java 3D [17]. Robotics Modeling and Simulation Platform (вовомоsP), otra plataforma virtual fue desarrollada por el grupo de automatización y robótica de la Pontificia Universidad Javeriana, en Cali, Colombia. Es una multiplataforma en 3D que soluciona el problema de la dinámica multicuerpo, al utilizar un enfoque basado en el cálculo automático de las propiedades de la masa de robot multicuerpo. También, permite modelar componentes físicos individuales y compuestos, todo con énfasis en robótica. Existen diversidad de laboratorios virtuales destinados a fines de ingeniería robótica, por ejemplo, el Virtual Cim Laboratory (vcimLAB), desarrollado en la Eastern Mediterranean University, en la República de Chipre, cuyo propósito es capacitar a los usuarios sobre los principios operativos de la fabricación integrada de computadoras (CIM) y sistemas de producción automatizadas que hacen uso de robots industriales y equipo de ensamble automatizado. Proporciona un entorno virtual interactivo en 3D y consiste en modelos de simulación virtual de hardware CIM, robots, máquinas y sistemas informáticos. El laboratorio de referencia consta de dos celdas de fabricación flexibles, incluyendo brazos de robot programables, una fresadora y varias piezas de equipos CIM. El entorno virtual de simulación representa muy claramente el laboratorio real. Los estudiantes pueden operar los modelos de simulación virtual, de acuerdo con los principios de funcionamiento en tiempo real $[16,17]$.

Otro laboratorio virtual a destacar es VirtualRobot, un software libre desarrollado en C++ por el Grupo de Robótica DISA-UPV (Valencia, España). Se trata de una aplicación gráfica de software diseñada a fin de que sea una plataforma general, flexible y abierta para aplicaciones de robótica. Fue diseñada en 1998 y es una herramienta útil en campos de robótica tales como robots industriales, control de robot móvil y detección de colisiones, entre otras. Además, puede simular máquinas multiaxiales como transportadoras, giradiscos, sistemas de sensores y robots móviles. Existen diversidad de aplicaciones virtuales con énfasis en robótica, por lo que también se puede mencionar al Virtual Laboratory for Robotics (VRL), desarrollado en la Escuela de Ingeniería Eléctrica de la Universidad de Belgrado, Serbia, y cuyo fin es mostrar las características principales de robots industriales. Contiene cinco configuraciones robóticas, las más comunes UMs2, Kuka R850, Kuka KR 1000 Titan, STAUbli Rx270 y eTF [16].

Además, se puede nombrar a USARSim como un simulador multirobot de código abierto, el cual se puede utilizar, tanto para educación, como para investigación. El desarrollo del simulador 
comenzó en la Universidad Carnegie Mellon y en la Universidad de Pittsburgh. USARSim es altamente extensible y configurable, permite a los usuarios agregar fácilmente nuevos sensores, o incluso modelar nuevos robots. Por su parte, el Cell Oriented Simulation of Industrial Robots (COSIMIR) es un paquete de software de emulación de robots, desarrollado por la Universidad de Dortmund en Alemania. Proporciona un entorno de aprendizaje virtual en el campo de la robótica. Su trabajo se basa en un complejo trabajo celular en ambientes reales simulados en 3D. Las celdas pueden contener modelos de robots Bosch Rexroth, Mitsubishi y Staubli. Todas las operaciones de movimiento y manipulación pueden ser simuladas con el fin de evitar colisiones y optimizar los tiempos. Por último, cabe mencionar a RoboLogix como un software de simulación robótica desarrollado por Login Design Inc (LDI), Canadá. Se diseñó con el propósito de emular aplicaciones robóticas del mundo real, e incluye un amplio rango de aplicaciones prácticas de robótica industrial basadas en cinco ejes, entre las que se encuentran soldadura, pintura y entornos personalizados, a fin de que el usuario pueda diseñar su propia aplicación [16].

Es importante mencionar algunos de los mundos virtuales que han surgido con el desarrollo de laboratorios virtuales. Entre estos se encuentran Second Life, un ambiente virtual online lanzado en el 2003 y desarrollado por Linden Labs. Este incluye edificios, islas y océanos; los usuarios pueden crear avatares y proporcionarles las características que deseen. Otros de estos conocidos mundos virtuales son RealXtend (comenzó en el 2007 y se basó, originalmente, en OpenSimular), así como OpenSimulator, Open Wonderland, Open Cobalt y United 3D [16].

Las aplicaciones que se han desarrollado para el campo de la medicina son diversas. Paquetes de software para la visualización de mundos dinámicos virtuales en 3D como Poser, Maya, 3D Studio, ZBrush, Blender, SecondLife, Avizo, Amira y Analyze permiten generar modelados virtuales animados en 3D, con el objetivo de diseñar cualquier tipo de figura, modelarla, tener control sobre los elementos que la conforman, así como de ayuda para reconstrucciones infográficas de escenas o para la representación de esquemas médicos o morfológicos relativos al cuerpo humano [18]. Entre estos se pueden nombrar simulación de quirófanos, cirugías y pruebas de fármacos en seres humanos [16].
En el campo automotriz, el modelo de simulación ha sido uno de los métodos de optimización más utilizados para el funcionamiento de grandes sistemas. Esto permite la creación de modelos virtuales de los procesos en los que se puedan explorar las consecuencias de ciertos cambios en ellos. Una de las aplicaciones para obtener resultados deseados, sin el uso de experimentación a gran escala y evitar situaciones de riesgos por errores en el diseño, es Tecnomatix Plant Simulation, diseñado para la creación del modelo, análisis de procesos y experimentación con simulación [19]. Este paquete de software permite la simulación y optimización de los sistemas organizativos y técnicos, así como los procesos de fabricación, los flujos de materiales y las operaciones logísticas [15].

Conforme se logran crear aplicaciones para la ejecución de procesos industriales, también se logra modelar las actividades de los operadores en 3D para el montaje en producción, con base en la información del flujo de trabajo y en diseños bidimensionales de las áreas de trabajo; en este contexto aparece Tecnomatix Jack [20].

En la actualidad, existen numerosos desarrollos de software para la simulación de la actividad humana ampliamente utilizados en la resolución de cuestiones de protección del trabajo en las empresas de diferentes industrias, tales como la automotriz y de manufactura $[15,20]$. Las más modernas herramientas de modelado son: Tecnomatix Jack, RAMSIS Automotive, DELMIA Ergonomics Task Definition, SAmmie y Santos Human [15].

En el campo de la ingeniería, una de las herramientas más comunes empleadas en la educación es el simulador [21]. Los simuladores se desarrollan bajo un modelo matemático que refleja, en mayor medida, el comportamiento del fenómeno físico simulado, con la dificultad que aún es compleja de la inclusión de todas las variables presentes en el fenómeno a estudiar.

Estos simuladores, desde la creación de las computadoras y el avance en las redes de comunicación, han sido de gran utilidad en el inicio de cursos como circuitos eléctricos y electrónicos para la formación de ingenieros mecánicos, de telecomunicaciones, de control, eléctricos y electrónicos. Bajo este ideal, se desarrollan entornos virtuales que, mediante lenguaje de programación gráfica, permiten realizar el diseño de sistemas dinámicos útiles para múltiples aplicaciones. Es así como la 
empresa National Instruments comienza su participación con la creación de LABVIEW para el tratamiento y procesamiento de señales, y expande sus alcances con instrumentación electrónica, automatización industrial y control [16, 21].

MatLab, un software matemático con un entorno de desarrollo interactivo, ha sido ampliamente usado junto con LABVIEW con el fin de crear diversas plataformas virtuales, ya que permite realizar control y automatización de múltiples procesos presentes en el campo de la ingeniería. Así, muchas universidades y grupos de investigación han elaborado nuevos entornos virtuales interactivos para el proceso de enseñanza-aprendizaje mediante la integración con hardware para la adquisición de datos $[16,22]$.

\subsection{Laboratorios virtuales en ingeniería eléctrica}

En el ámbito de la ingeniería eléctrica, la tecnología es un elemento que propone nuevos enfoques metodológicos para la enseñanza de circuitos eléctricos, electrónica básica, electrónica de potencia, máquinas eléctricas, líneas de transmisión, sistemas de potencia, subestaciones y protecciones. Muchos de estos cursos se han limitado al uso de herramientas "planas" de e-learning, las cuales generalmente inhiben la interactividad a la recepción y envío de texto plano e información multimedia. Por lo anterior, la tecnología con visualización en $3 \mathrm{D}$ se ha convertido en una alternativa válida para la comprensión de temáticas de aprendizaje y mejorar la experiencia del estudiante en su paso por la asignatura, sin dejar de lado la interacción con simuladores en 2D. Este enfoque permite al aprendiz dimensionar su objeto de estudio, comprender un hecho real al que no tiene acceso sino hasta terminar su formación profesional, y experimentar con una metodología que se aproxima a un entorno real [23].

El enfoque pedagógico de proyectos basados en ambientes virtuales en 2D y 3D para cursos teórico-prácticos, permite estimular al estudiante a indagar y experimentar, a obtener sus propios análisis y concluir sobre su experiencia en el aula virtual, de manera que el trabajo autónomo y cooperativo incrementa significativamente [24].

Cursos propios del programa de Ingeniería Eléctrica han intentado mostrar un modelo virtual de aprendizaje basado en simuladores, los cuales han alcanzado un desarrollo en 2D y 3D para implementar laboratorios de circuitos básicos en DC y AC, máquinas eléctricas y electrónica de potencia, entre otras. Cursos - e. g. en campos electromagnéticos-, han utilizado software con módulos electrostáticos y magnetostáticos para el cálculo de campos eléctricos y magnéticos generados por un conductor al circular una corriente eléctrica. COMSOL MULTIPHYSIC [25], un software basado en el método de elementos finitos, ha sido ampliamente usado para la comprensión de conceptos de campos electrostáticos como la ley de Coulomb, la ley de Gauss, la ley de Biot-Savart y la ley de circuitos de Ampere [26].

En sistemas eléctricos de potencia, líneas de transmisión y protecciones eléctricas, el uso de simuladores se ha enmarcado en 2D con el cálculo de corrientes, voltajes, flujos de potencia, análisis de fallas (corriente de cortocircuito), armónicos y coordinación de protecciones, entre otros. Uno de los simuladores usados en la industria eléctrica para el análisis de sistemas de potencia y sistemas de distribución es el Electrical Transient Analyzer Program (ETAP), que, además de su enfoque en sistemas de alta tensión, es una opción para el diseño e instalación de sistemas fotovoltaicos y eólicos. Al igual que ETAP, el Power System Analysis and Engineering (DIGSILENT) y NEPLAN poseen características de simulación similares, por lo que constituyen variedad de opciones al momento del diseño, el análisis, la planeación, la optimización y la operación de sistemas eléctricos.

Otros simuladores que se pueden mencionar son Power Line Systems-Computer Aided Design and Drafting (PLS-CAAD) y DLTCAD; estos para el diseño de líneas de transmisión que utilizan un sofisticado modelo de ingeniería tridimensional con vistas de perfil, vistas de planta, elementos en 2D y 3D. Dentro de la gran variedad de aplicaciones virtuales utilizadas en ingeniería eléctrica, se encuentran distintas herramientas virtuales, como, por ejemplo, el simulador de subestaciones eléctricas creado por estudiantes de la Universidad Distrital Francisco José de Caldas, cuyo desarrollo, experimentación y validación se presentará más adelante [27].

La Universidad Distrital Francisco José de Caldas, en su propósito de innovar los procesos de enseñanza, busca incentivar el uso de espacios y entornos virtuales como un apoyo pedagógico en 
las diferentes asignaturas de los proyectos curriculares existentes en la universidad. En los últimos años, el uso de MOODLE, una plataforma virtual multipropósito, se ha implementado a fin de que los profesores y estudiantes puedan compartir material de apoyo (videos, fotos, documentos digitales, etc.), además de permitir una comunicación bidireccional global, es decir, que los estudiantes puedan expresar sus dudas y, tanto el docente, como sus compañeros, puedan contestar. En este sentido, los diversos grupos de investigación se están enfocando en el desarrollo de aplicaciones virtuales que sirvan como una herramienta complementaria a la formación académica. En el programa de Ingeniería Eléctrica de la Facultad de Ingeniería de la Universidad Distrital Francisco José de Caldas, se han desarrollado dos herramientas virtuales para el estudio de subestaciones eléctricas: un simulador soportado en un ambiente en 2D para la enseñanza de maniobras eléctricas y conceptos teóricos, cuyo desarrollo se encuentra en el trabajo de grado Aplicación educativa para maniobras y estudio de subestaciones eléctricas, de Astrid Carolina Herrera-Díaz, y cuyo director es el ingeniero William Alfonso RiañoMaldonado. Este proyecto de grado se fundamenta en la creación de un módulo interactivo para realizar maniobras eléctricas en diferentes configuraciones y afianzar conceptos teóricos.

El desarrollo más reciente es un simulador modelado en 3D que surge como un trabajo conjunto de los proyectos curriculares de Ingeniería Eléctrica e Ingeniería de Sistemas, de la Facultad de Ingeniería, y se encuentra en el trabajo de grado Desarrollo de entornos virtuales interactivos de configuraciones de subestaciones eléctricas de barra simple y doble barra, como material de apoyo educativo, de Juan José Carvajal-Villalobos y William Fabián Ardila-Guzmán, y cuyos directores fueron la ingeniera Diana Stella García-Miranda, en colaboración con el ingeniero William Alfonso Riaño-Maldonado. La investigación se fundamenta en la creación de modelos tridimensionales interactivos para las configuraciones de barra simple y barra doble de subestaciones eléctricas, como herramienta pedagógica para la comprensión del tema de maniobras eléctricas, y así contribuir a reducir los altos índices de repitencia de los estudiantes en la asignatura.
La asignatura de subestaciones eléctricas requiere de un componente técnico que permita al estudiante conocer la operación y el mantenimiento de este tipo de instalaciones eléctricas, de manera que surge la necesidad de experimentar en un ambiente real, y de esta forma, fortalecer el conocimiento teórico adquirido en un aula de clase. Sin embargo, estas instalaciones suelen ser de acceso restringido, dados los riesgos asociados a los elevados niveles de tensión que allí se manejan, motivo por el cual es complejo lograr la experimentación en este campo. Las personas que operan subestaciones eléctricas deben demostrar un nivel de preparación técnica que les permita evaluar los riesgos del manejo de equipos en alta tensión, minimizando las posibilidades de sufrir un accidente durante la operación. Esa preparación técnica no se logra adquirir en un aula de clase, pues es difícil que los estudiantes de la asignatura logren interactuar y vivenciar estos procesos, razón por la cual se decidió incursionar entre los estudiantes de Ingeniería de Sistemas el logro del desarrollo de un simulador que permitiera suplir la necesidad de experimentación en el requerimiento de una formación técnica, además de lograr una mayor comprensión en temas de operación y mantenimiento con enfoque en maniobras eléctricas que, como se mencionó anteriormente, era una de las principales causas de pérdida y repitencia de los estudiantes en la asignatura.

\section{4 Índices de repitencia en subestaciones eléctricas}

La revisión de evaluaciones presentadas en la asignatura de subestaciones eléctricas para el periodo 2008-2012 permitió revelar que uno de los temas más difíciles de asimilar por parte de los estudiantes era realizar las diferentes maniobras eléctricas, de acuerdo con las configuraciones existentes en este tipo de instalaciones. Un estudio realizado muestra cifras, en porcentaje, de estudiantes que perdieron la asignatura por semestre entre el 2006 y el 2016. La tabla 1 expone dichos porcentajes y se representan en la figura 1. 
Tabla 1. Índices de repitencia de la asignatura subestaciones eléctricas del 2006 al 2016

\begin{tabular}{|c|c|}
\hline Periodo & Estudiantes que no aprobaron la asignatura \\
\hline 2006-III & $20 \%$ \\
\hline 2007-I & $50 \%$ \\
\hline 2007-III & $38,1 \%$ \\
\hline 2008-I & $73,1 \%$ \\
\hline 2008-III & $35,3 \%$ \\
\hline 2009-I & $71 \%$ \\
\hline 2009-III & $56,5 \%$ \\
\hline 2010-I & $39,1 \%$ \\
\hline 2010-III & $32,4 \%$ \\
\hline 2011-I & $51,3 \%$ \\
\hline 2011-II* & $6 \%$ \\
\hline 2011-III & $34,6 \%$ \\
\hline 2012-I & $25,4 \%$ \\
\hline 2012-III & $20,8 \%$ \\
\hline 2013-I & $15,6 \%$ \\
\hline 2013-III & $37,5 \%$ \\
\hline 2014-I & $43,5 \%$ \\
\hline 2014-III & $75,6 \%$ \\
\hline 2015-I & $33,3 \%$ \\
\hline 2015-III & $32,5 \%$ \\
\hline 2016-I & $8,7 \%$ \\
\hline
\end{tabular}

${ }^{*}$ Este período fue un vacacional

Fuente: Oficina Asesora de Sistemas (OAs)
La figura 1 muestra las variaciones, en porcentaje, de estudiantes repitentes en los diferentes periodos académicos, sin tender a un incremento o decremento. Los datos presentados hasta este momento, pueden ser resumidos a través del uso de un histograma u otros métodos tabulares y gráficos útiles, con el fin de lograr una interpretación adecuada.

Mediante una distribución de frecuencias, es posible conocer el número de estudiantes que han cursado la misma asignatura entre una y cinco veces, del 2006 al 2012, y representarlos mediante un histograma. Con los datos expuestos en la tabla 2 fue posible construir el diagrama de barras de la figura 2.

Tabla 2. Índices de repitencia de la asignatura subestaciones eléctricas del 2006 al 2012

\begin{tabular}{|c|c|}
\hline $\begin{array}{c}\text { Número de veces } \\
\text { (Repitencia) }\end{array}$ & Cantidad de estudiantes \\
\hline 1 & 116 \\
\hline 2 & 31 \\
\hline 3 & 6 \\
\hline 4 & 3 \\
\hline 5 & 2 \\
\hline
\end{tabular}

Fuente: Oficina Asesora de Sistemas (OAs)

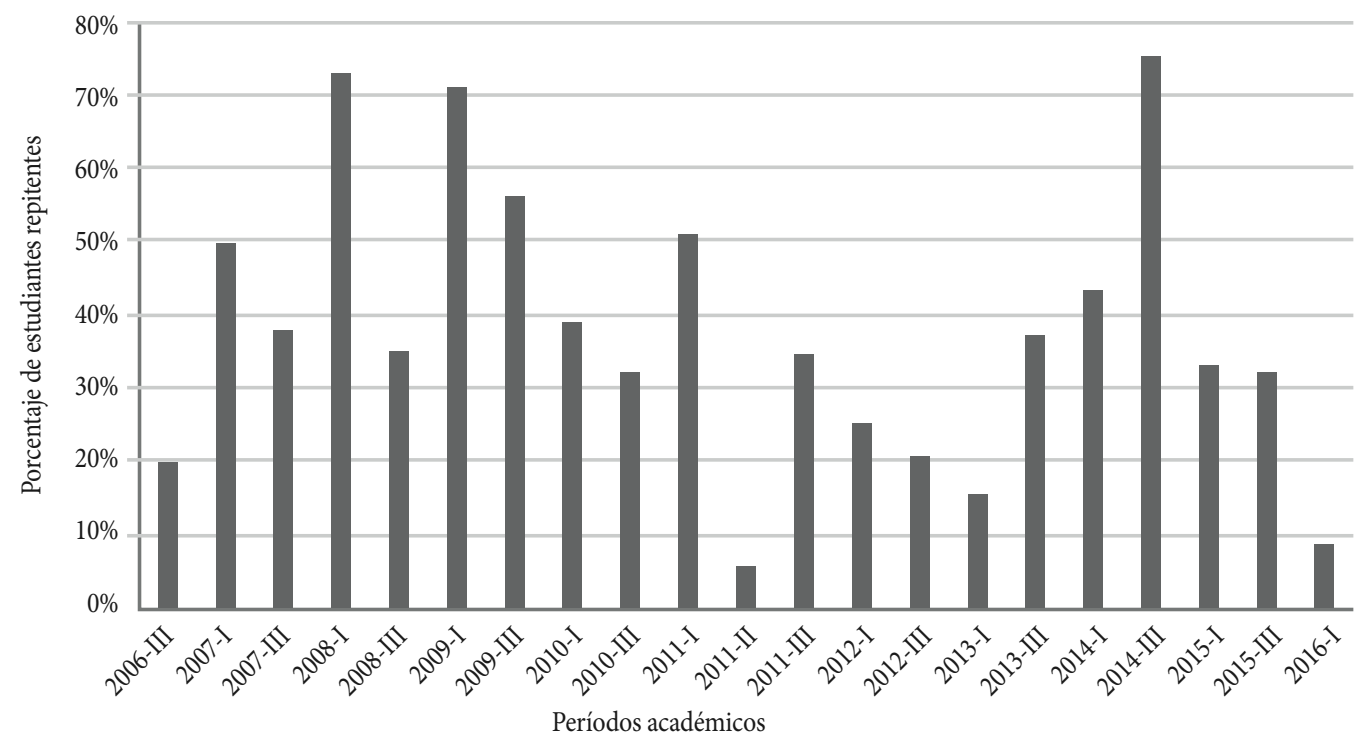

Figura 1. Indicadores de repitencia en subestaciones eléctricas para el periodo del 2006 al 2016

Fuente: elaboración propia con base en información de la Oficina Asesora de Sistema (oAs) 
El histograma muestra que el mayor número de estudiantes que repitieron la asignatura lo hicieron una sola vez, y solo dos personas la repitieron cinco veces del 2006 al 2012. Sin embargo, el hecho de cursar de nuevo la asignatura, aunque sea una sola vez, generó preocupación entre el profesorado, por lo que se buscó determinar las causas de repitencia para hallar soluciones a largo plazo. Una de ellas fue el desarrollo de un simulador, tal y como se mencionó anteriormente.

Un promedio de los valores de la tabla 1 correspondiente a un $38 \%$, permitiría observar en la gráfica de la figura 1 que son varios los periodos que están por debajo de este valor, e incluso en un porcentaje mucho menor. Una investigación profunda identificaría las causas que conducen a mostrar estas cifras en semestres como el 2006III, 2011-III, 2012-I, 2012-III, 2013-I y 2016-I, lo cual no se puede atribuir a la implementación de nuevas metodologías de aprendizaje, o a factores externos tales como anormalidad académica. Esta condición afecta la culminación exitosa del curso y la evaluación de todos los temas propuestos en el contenido programático. Por tal motivo, se propone, en algunas ocasiones, realizar currículo alterno, lo que flexibiliza la evaluación del curso y permite un mayor aprovechamiento de las horas de trabajo autónomo. Si se observa la figura 1 , los periodos del 2011-I al 2013-I inician con un $6 \%$, incrementan a un
$34,6 \%$ y, a partir de este punto, empiezan a disminuir, para terminar en un 15,6 \% en el 2013-I. Para el 2011II se realizó un curso vacacional, en el que el índice de repitencia fue únicamente del $6 \%$. Esto se debe a que los estudiantes se encuentran dedicados únicamente a esta asignatura, aumentando el tiempo de estudio autónomo, sin estrés y preocupaciones de responder por trabajos y evaluaciones de otras asignaturas. Para el 2011-III, 2012-I y 2013-I se presentó anormalidad académica, por lo que las cifras de indicadores de repitencia tendieron a disminuir, aclarando que este no es el único factor influyente en las cifras, pero sí el que más incidencia tuvo sobre las mismas.

\section{Metodología}

Se resalta la importancia del uso de las Tic en el proceso de enseñanza-aprendizaje, junto con una revisión de ambientes virtuales en diferentes campos tales como medicina, industria automotriz, ciencias e ingenierías con enfoque en ingeniería eléctrica, mediante el uso de bases de datos como Scopus, Web of Science e IEEE, entre otras. El simulador de subestaciones eléctricas se presenta con las configuraciones de barra simple y barra doble, simulación de maniobras (para mantenimiento, reparación o puesta en operación de los equipos), evaluación de la maniobra y descripción de los equipos presentes

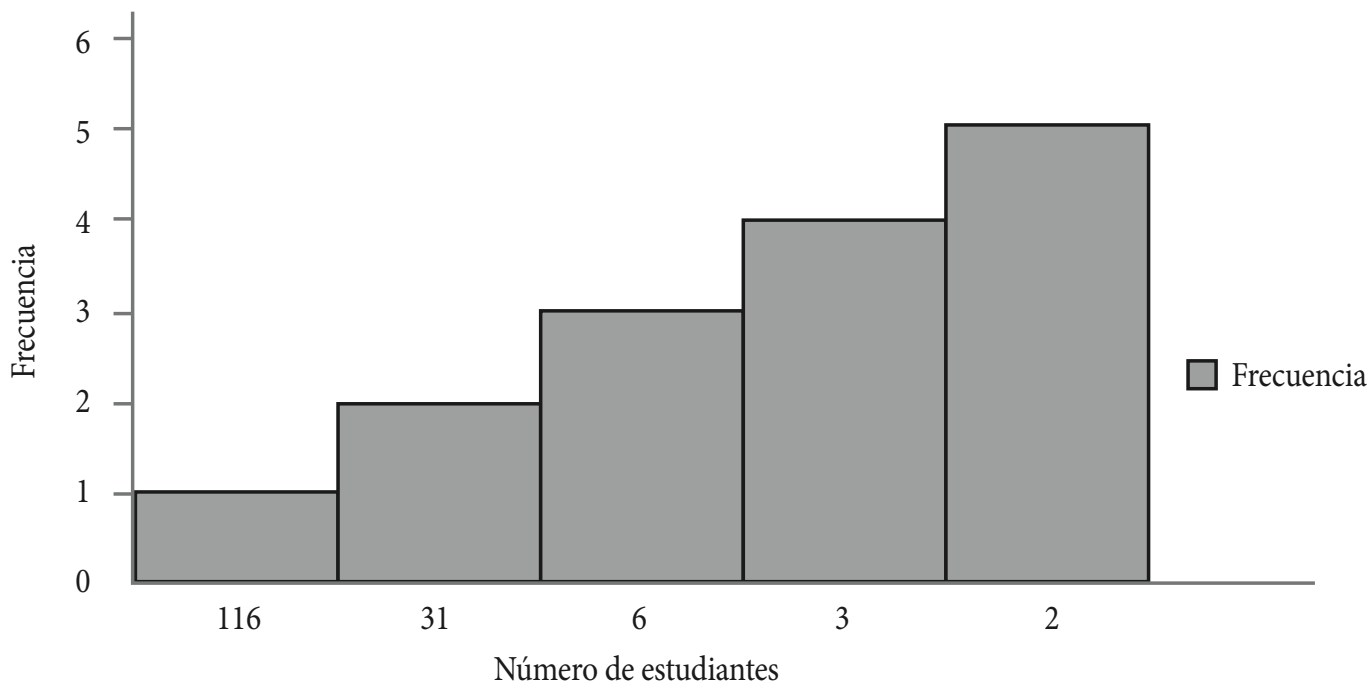

Figura 2. Histograma para índices de repitencia

Fuente: elaboración propia del autor con base en información de la Oficina Asesora de Sistema (OAs) 
en la instalación a través de su interfaz gráfica, con un breve análisis sobre su aporte a la asignatura de subestaciones eléctricas. Además, se exponen datos de cifras de repitencia en tablas, histogramas o diagramas de barra como justificación al desarrollo de este proyecto, y se realiza la propuesta de ampliar la herramienta de simulación con módulos de evaluación respecto a maniobras y módulos relacionados con el diseño (coordinación de aislamiento, dimensionamiento de transformadores y conductores, sistema de puesta a tierra, sistemas de apantallamiento), mediante la creación de nuevas interfaces gráficas que incluyan las diferentes configuraciones de subestaciones eléctricas e imágenes que permitan ilustrar al usuario, así como mostrar todo el conocimiento con respecto a la ejecución de maniobras y el diseño de subestaciones.

\subsection{Simulador de subestaciones}

Las subestaciones eléctricas permiten la trasformación de algunos parámetros eléctricos - a fin de adecuarlos al transporte y la distribución de la energía eléctrica一, tales como amplitud, frecuencia y forma de onda de las señales de tensión y corriente, además de garantizar la continuidad en el suministro. Debido a los altos niveles de tensión presentes en este tipo de instalaciones, estas se convierten en un entorno peligroso para una persona que no posea los conocimientos en el área, de manera que son espacios de acceso restringido y limitan la interacción del estudiante con una subestación real cuando se encuentra cursando las asignaturas de sistemas de potencia, subestaciones eléctricas y protecciones, fundamentales en la formación de un ingeniero electricista. Una solución a esta problemática está en el apoyo de la nuevas tecnologías, al crear entornos de simulación que permitan a los estudiantes consolidar los conocimientos teóricos vistos en clase. Así, por ejemplo, podrán visualizar físicamente los equipos de patio de acuerdo con cada configuración, interactuar con los diferentes equipos de maniobra necesarios para desconectar parte del sistema eléctrico de potencia, ya sea para realizar mantenimiento en algún elemento del sistema, aislar una falla o emular cualquier otra situación real que se pueda presentar en una subestación.

El proyecto curricular de Ingeniería Eléctrica, con el apoyo de Ingeniería de Sistemas de la Facultad de Ingeniería de la Universidad Distrital Francisco José de Caldas, desarrolló un simulador de subestaciones eléctricas con un enfoque pedagógico en el estudio de maniobras eléctricas, asociado a barra sencilla y barra doble (figuras 3 y 4 ). El simulador está basado en el "aprender haciendo", es decir, en que la mayor parte del aprendizaje depende de la experiencia. El simulador es una propuesta que utiliza herramientas virtuales que permiten a los estudiantes tener acercamientos a los diferentes elementos de maniobra y medida, al simular una subestación eléctrica en operación.

El simulador se encuentra en su primera versión, se implementó en Python para la arquitectura lógica, y Blender 2,70 para el desarrollo de la interfaz gráfica. El uso de Blender y Python se justifica en que son de libre acceso, tanto el archivo ejecutable, como el código fuente, y se han diseñado para ser compatibles con diferentes sistemas operativos, de modo que las aplicaciones creadas en estos entornos de desarrollo tengan la posibilidad de ser difundidas a nivel mundial, con el propósito de que otros usuarios y/o desarrolladores puedan mejorar el código existente y agregar más configuraciones al simulador. Particularmente, Python permite que los desarrollos sean, en términos generales, mucho más fáciles de leer y ocupen menos líneas de código, en comparación con otros lenguajes de programación [5].

El simulador se desarrolló e implementó bajo la metodología ágil de desarrollo de proyectos Scrum, contexto en el cual se proyectan cuatro grandes iteraciones (Sprints) durante el ciclo de desarrollo. La primera abarca el diseño y modelado de la subestación eléctrica, es decir, los elementos constituyentes de la misma asociando sonidos, efectos especiales y elementos del medio ambiente. La segunda integra las interfaces de usuario y la animación de componentes de la subestación. La tercera comprende la programación y lógica de eventos para las distintas interacciones del usuario con el simulador. Por último, la cuarta engloba validaciones y pruebas, en las que se realizan los últimos ajustes previos a la compilación y generación de entregables del simulador [5]. Estos procesos iterativos se muestran con mayor claridad en la figura 5 que integra los procesos a la producción en 3D.

El proceso que se presenta a continuación es una metodología adoptada y descrita por los usuarios y desarrolladores del software según [5], y se menciona en el presente artículo como una manera de explicar las estrategias y/o metodologías que se adoptaron a fin de lograr el desarrollo del simulador de subestaciones eléctricas. 


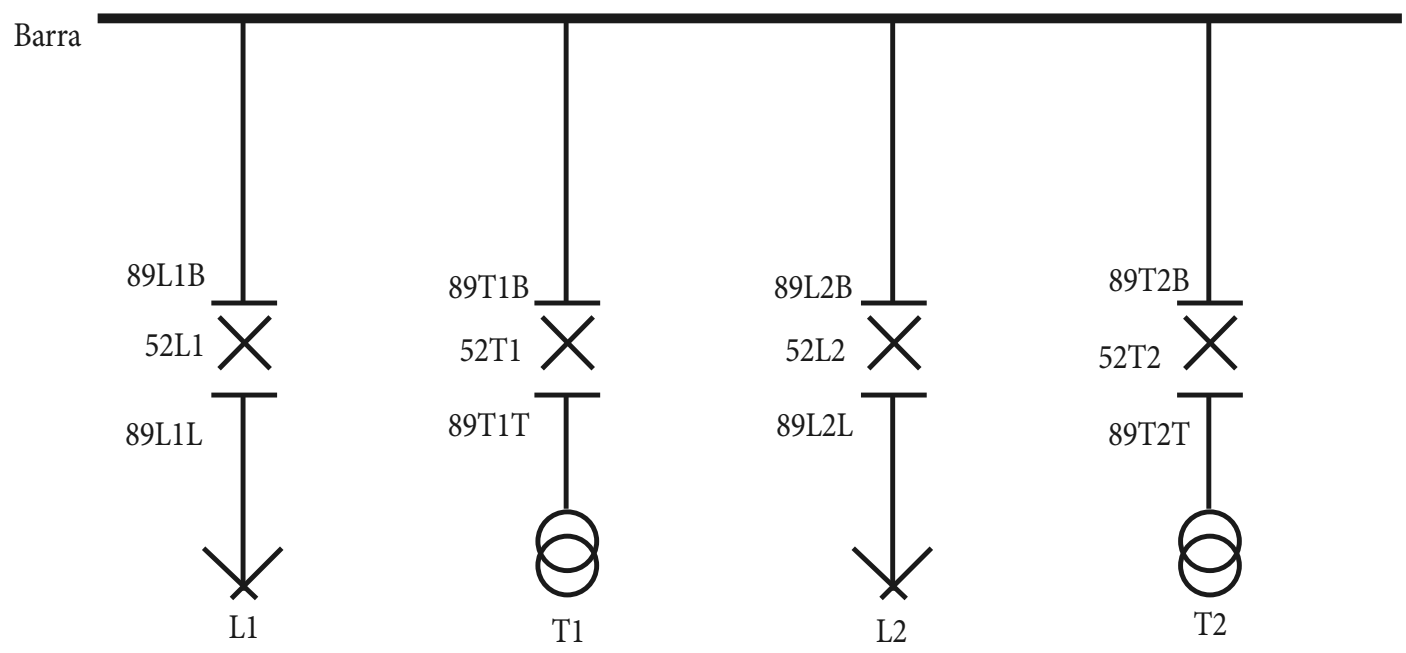

Figura 3. Configuración de barra simple con dos líneas y dos transformadores Fuente: [5]

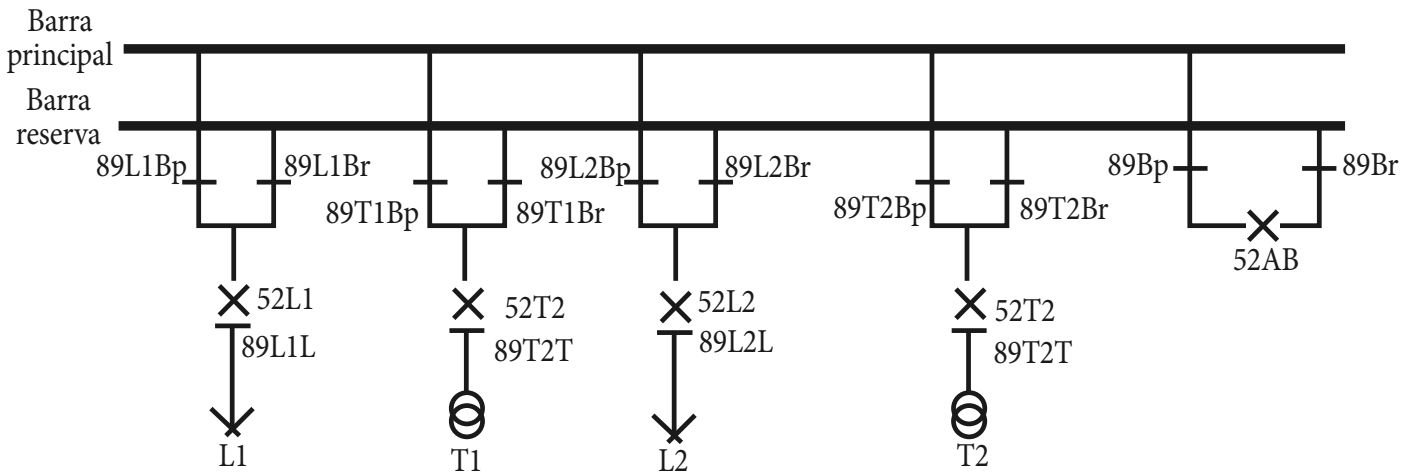

Figura 4. Doble barra de $145 \mathrm{Kv}$ con tres líneas y dos transformadores Fuente: [5]

Los desarrolladores del software hicieron uso de los principios del manifiesto ágil para todas y cada una de las fases que componen el ciclo de vida del simulador de subestaciones eléctricas. Tales principios establecen satisfacción al cliente con la entrega temprana y continua del software con valor, aceptación al cambio de los requisitos -incluso, en etapas tardías del desarrollo-, y trabajo continuo en conjunto con los responsables del negocio y desarrolladores, entre otros principios [5]. Con lo anterior, se implementó la metodología de desarrollo ágil Scrum de tipo iterativo incremental, compuesta de iteraciones llamadas "Sprint", de aproximadamente un mes de duración, en la cual se llevan a cabo las tareas que son necesarias para lograr una o varias funcionalidades, las cuales se planean al inicio [5].
En la metodología Scrum se definen tres roles: el dueño del producto, quien establece y da prioridad a los distintas funcionalidades que se desea en el producto; el equipo de desarrollo, quienes desarrollan e implementan las distintas funcionalidades que caracterizan al proyecto; $y$ el ScrumMaster, a cargo de gestionar y facilitar la ejecución de los procesos [5]. Es así que los desarrolladores establecieron su marco de trabajo mediante Scrum.

Este esquema de trabajo empieza por la definición de roles [29]. Cada Sprint representa una iteración y finaliza en un incremento del producto "Terminado". La definición de "Terminado" se concretará en la reunión de planificación en cada proceso, implicando al equipo y al dueño de producto. 


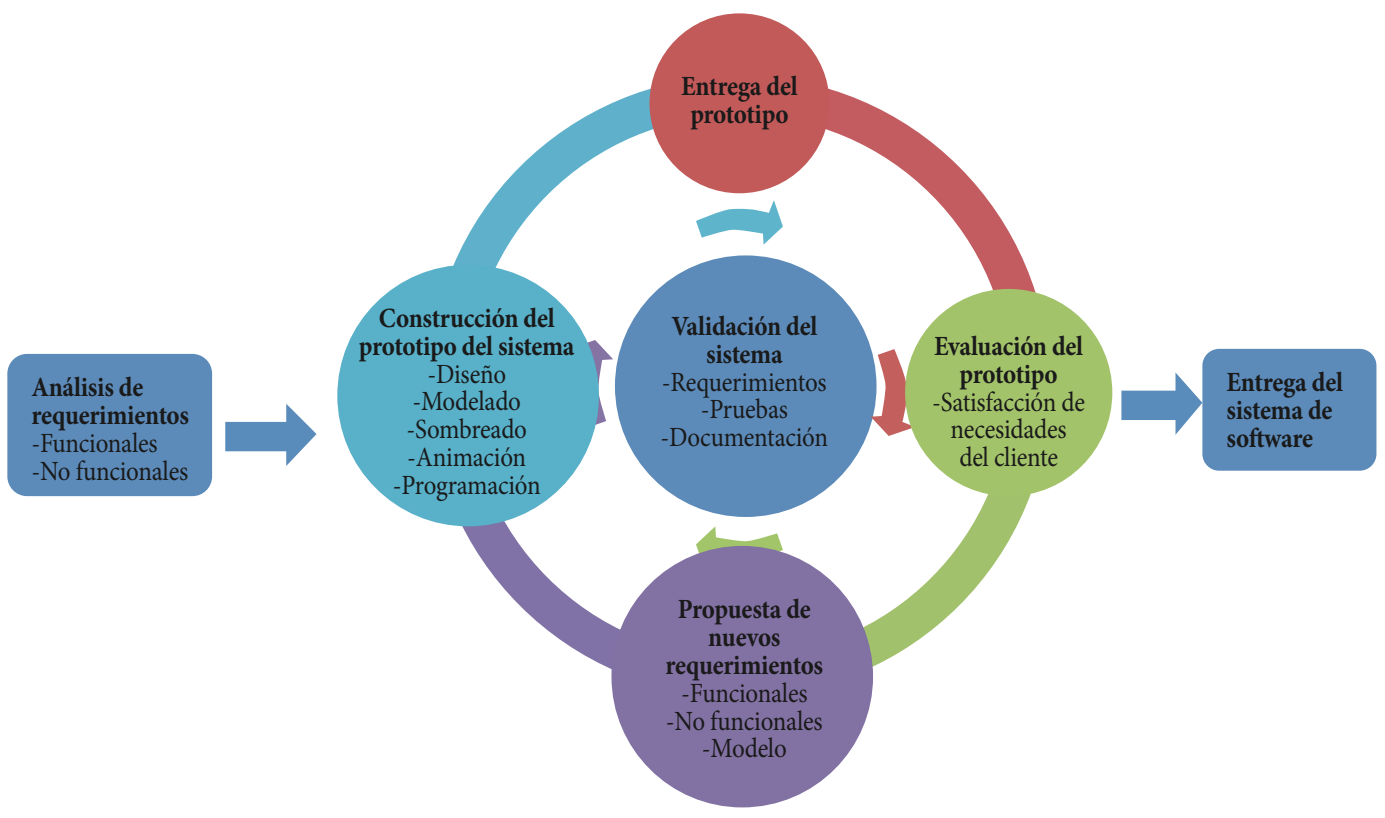

Figura 5. Metodología ágil de desarrollo de proyectos Scrum para el simulador de subestaciones eléctricas Fuente: [5]

En la reunión de planificación se establece el objetivo, y se describen "historias" que son requisitos funcionales descritos en lenguaje común (proveniente del llador a fin de elaborar la pila de la iteración. La pila

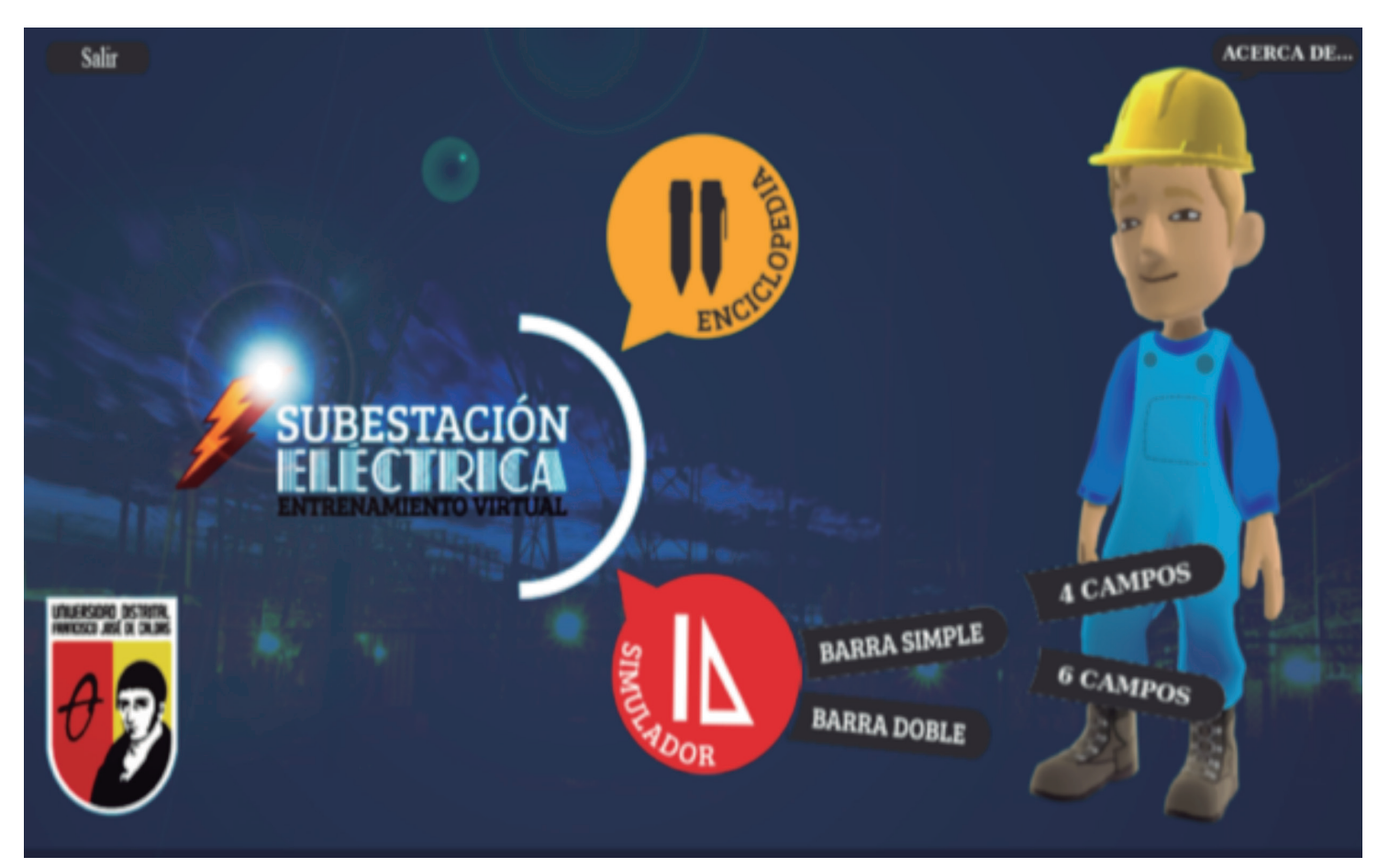

Figura 6. Menú de inicio del simulador de subestaciones

Fuente: [5] cliente). Además, se despejan dudas y se definen prioridades. Esta información la trata el equipo desarro- 
la constituyen las historias que el equipo se compromete a desarrollar a lo largo del proceso. Durante el transcurso de cada fase, cada una de las cuales tiene una duración de un mes (aunque puede variar entre dos semanas y dos meses, según el modelo), se llevará a cabo al inicio de cada día de trabajo una reunión de 15 minutos entre los miembros del equipo, con el propósito de mirar en retrospectiva lo que se ha hecho el día anterior y lo que se hará en ese día, distribuyéndose cada una de las tareas en el orden y prioridad que el equipo estime conveniente. Las tareas son los trabajos que es necesario realizar para satisfacer la condición de "Terminado" de una historia. Por último, al terminar el periodo de cada Sprint, se realiza la presentación del incremento, se hacen sugerencias y se despejan dudas. Este proceso se repite de manera iterativa hasta que el producto final satisface todos los requerimientos propuestos por el dueño de producto, y una vez se cumplan todos y cada uno de los objetivos establecidos en el proyecto [5].

El desarrollo del proyecto se describe en cuatro etapas, con una duración estimada según la siguiente enumeración [5]:
- Sprint 1. Diseño y modelado de subestación eléctrica: un mes.

- Sprint 2. Implementación de interfaces y animaciones: un mes.

- Sprint 3. Programación y lógica de eventos: dos meses.

- Sprint 4. Validación y pruebas: dos meses.

El simulador cuenta con una serie de criterios que se establecieron durante la selección de herramientas para su desarrollo. Tales criterios contemplan: ser un software libre; usarse para cualquier propósito y sin licenciamiento, orientado al desarrollo de contenidos en 3D; permite complementar su desarrollo con modelamiento en 3D, una interfaz de programación fácil de integrar e implementar; y robustez, es decir, es lo suficientemente robusto para implementar nuevas características y funcionalidades [5]. Actualmente, el simulador cuenta con dos entornos (figura 6), una enciclopedia en la que se muestra una lista de elementos típicos encontrados en una subestación, así como una breve descripción del elemento, y un modelo en 3D a escala, con el cual el usuario pueda interactuar y

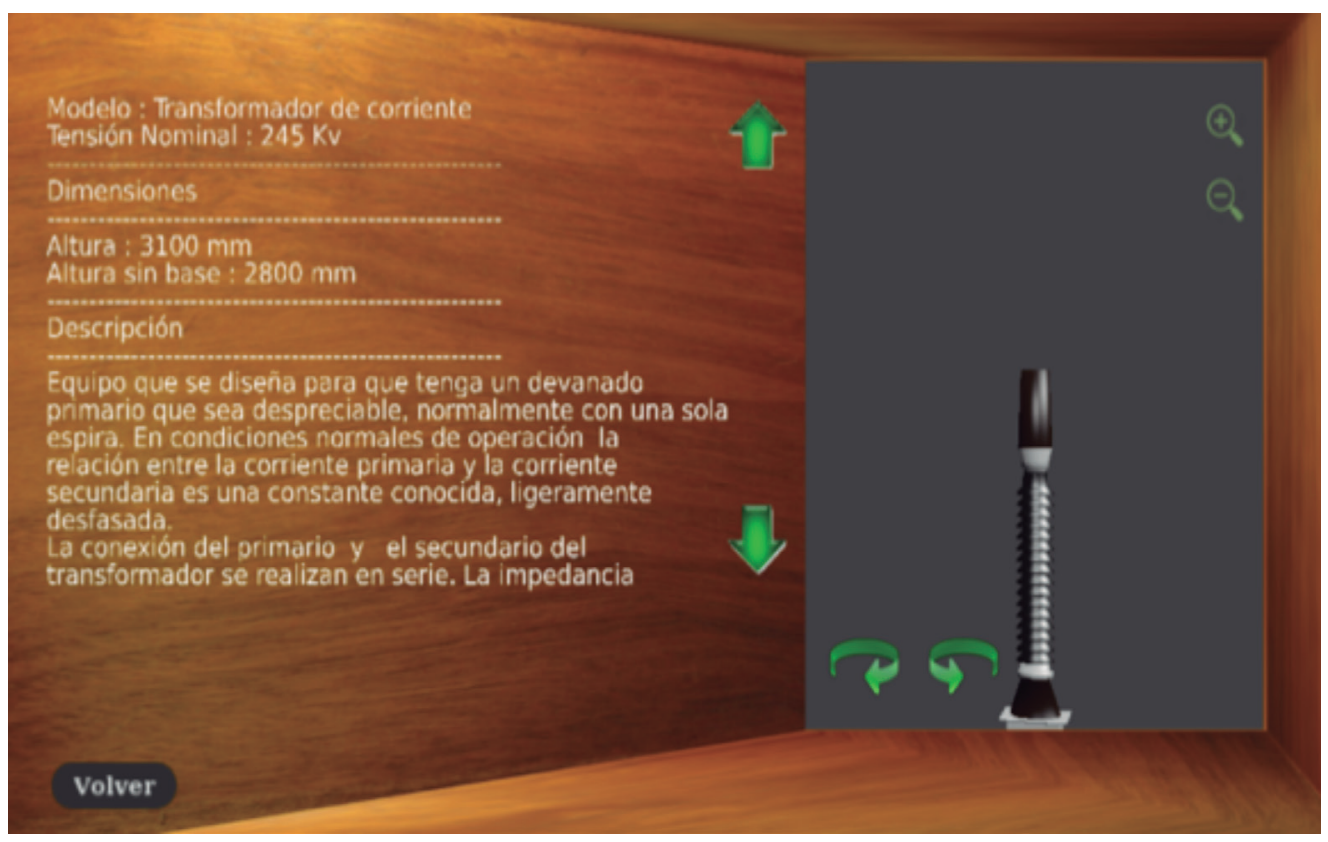

Figura 7. Elementos de patio con modelado en 3D. Enciclopedia CT Fuente: [5] 
ver detalladamente el elemento, así como familiarizarse con él, tal como se muestra en la figura 7.

El segundo entorno es el de simulación, el cual cuenta actualmente con dos configuraciones: barra sencilla ybarra doble (figuras 3 y 4). Cada configuración permite establecer entre cuatro o seis campos, una vez seleccionada la configuración y el número de campos se muestra un mímico (mapa de simulación) de la subestación que permite modificar el estado operativo de los elementos de maniobra (figura 8).

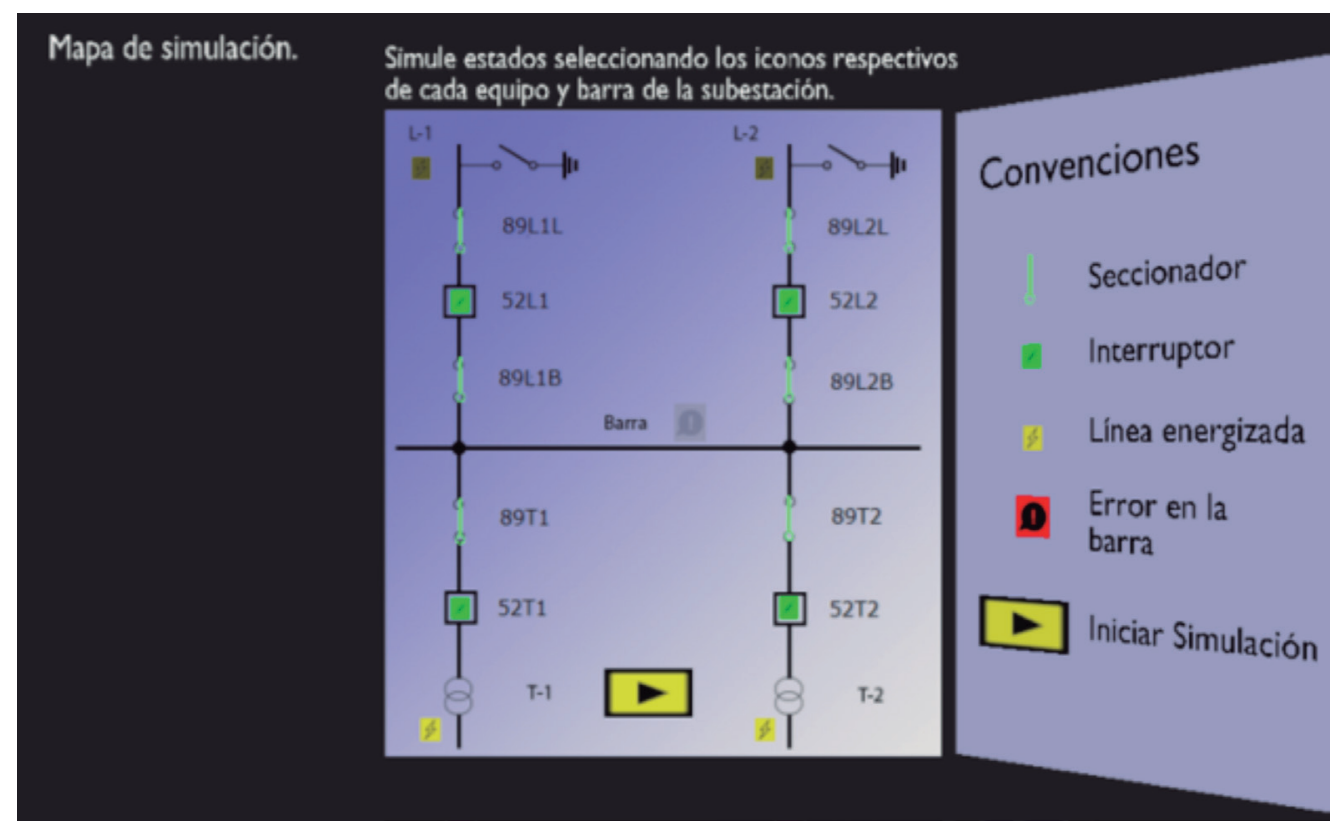

Figura 8. Mapa de simulación (mímico) de la subestación eléctrica para barra sencilla con cuatro campos

Fuente: [5]

Por último, el usuario ingresa al entorno de simulación, en el que se muestra una representación en 3D de una subestación con la configuración, el número de campos y el estado operativo de los elementos de maniobra previamente seleccionados (figura 9). En este entorno se puede interactuar con los equipos de maniobra como si se encontrara en una subestación real; en caso de realizar alguna maniobra que sea indebida, el simulador mostrará la imagen de un operario que ha sufrido una descarga eléctrica, acompañado de un sonido agudo y se indicará al usuario que ha realizado una operación incorrecta, se cargará nuevamente el menú de inicio, y se ofrece la posibilidad de seleccionar una nueva configuración.

A lo largo de su desarrollo, el simulador de subestaciones ha tenido diferentes mejoras. Después de su desarrollo inicial, por parte de Juan José Carvajal-Villalobos y William Fabián Ardila-Guzmán, estudiantes de Ingeniería de Sistemas, se detectaron algunos errores de programación en la configuración de doble barra, puesto que al realizar la maniobra de pasar un circuito de un barraje al otro, realizando la maniobra de forma correcta, la simulación terminaba indicando que se había realizado una maniobra de forma inadecuada o peligrosa. Por otra parte, tanto en la tendencia americana, como en la tendencia europea, los nombres de los elementos de maniobra están preestablecidos para no causar confusiones al ingeniero electricista en el momento de realizar una maniobra; se detectó que los nombres con los que se mencionaban los diferentes elementos de maniobra de las configuraciones existentes en el simulador no correspondían con ninguna tendencia.

Los estudiantes David Antonio Riveros-Hernández y Carlos Alfredo Bonilla-Castro, estudiantes de Ingeniería Eléctrica de la Facultad de Ingeniería de la Universidad Distrital, corrigieron los errores mencionados anteriormente, exaltando la importancia de este simulador como apoyo a la formación de ingenieros electricistas.

El simulador de subestaciones, hasta la fecha de publicación del presente artículo, cuenta con dos 


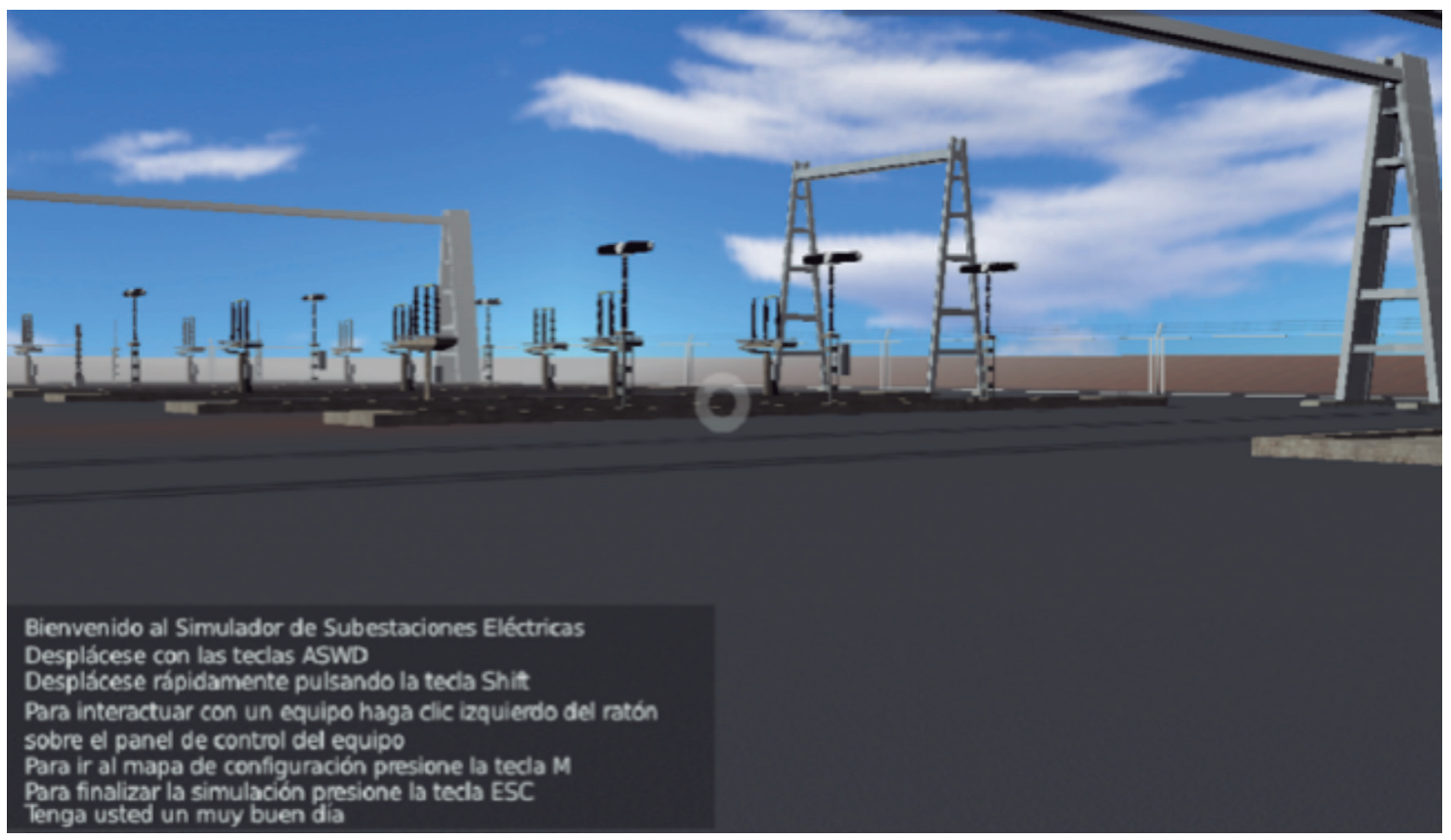

Figura 9. Entorno 3D de simulación de una subestación eléctrica Fuente: [5]

configuraciones: barra sencilla y barra doble. Aún está en proceso de desarrollo, y el objetivo es que estudiantes que cursen la materia de subestaciones eléctricas, por primera vez, agreguen elementos que enriquezcan el simulador y sea de mayor utilidad a los estudiantes que están por cursar la asignatura. Esta filosofía se ha llevado a cabo gracias a William Riaño-Maldonado y Diana Stella García-Miranda, docentes de Ingeniería Eléctrica de la Universidad Distrital, con el apoyo de los estudiantes, quienes creen firmemente que no todos aprenden de la misma forma y buscan recursos como la virtualidad con el propósito de ayudarlos a desarrollar las competencias necesarias para aprobar las diferentes asignaturas.

Dentro de los aspectos destacables del simulador se encuentran:

- Todos los elementos que presenta el simulador son representados por los materiales reales, fotografiados de una subestación real e implementados en cada uno de los objetos (realidad aumentada).

- El simulador cuenta con sonidos reales, tales como el sonido de los fenómenos físicos, algunos producidos por el efecto corona y la vibración de los trasformadores de potencia, apertura $\mathrm{y}$ cierre de interruptores.
- Simula el desplazamiento y/o rotación de los seccionadores cuando realizan apertura o cierre.

La ventaja del simulador contempla conocer los diferentes equipos de patio mediante su visualización en 3D, a fin de afianzar los conocimientos adquiridos en clase. La reproducción de sonidos tales como efecto corona, arco eléctrico, apertura y cierre de seccionadores e interruptores, pretenden hacer sentir al estudiante en un entorno real.

\subsubsection{Simulación}

La plataforma virtual tiene como objetivo mejorar las competencias de los estudiantes en el área de subestaciones eléctricas, mediante procesos de interacción que los lleven a consolidar los conocimientos adquiridos; por tal motivo, se presenta una simulación con el fin de detallar este proceso de aprendizaje, ya que es importante mostrar cómo podría ejecutarse una maniobra en el simulador de subestaciones eléctricas. Dentro de las posibilidades de simulación, se mostrará el mantenimiento de un interruptor para la configuración de doble barra con cuatro campos, partiendo de condiciones normales de operación, tal como se indica en la figura 10. 


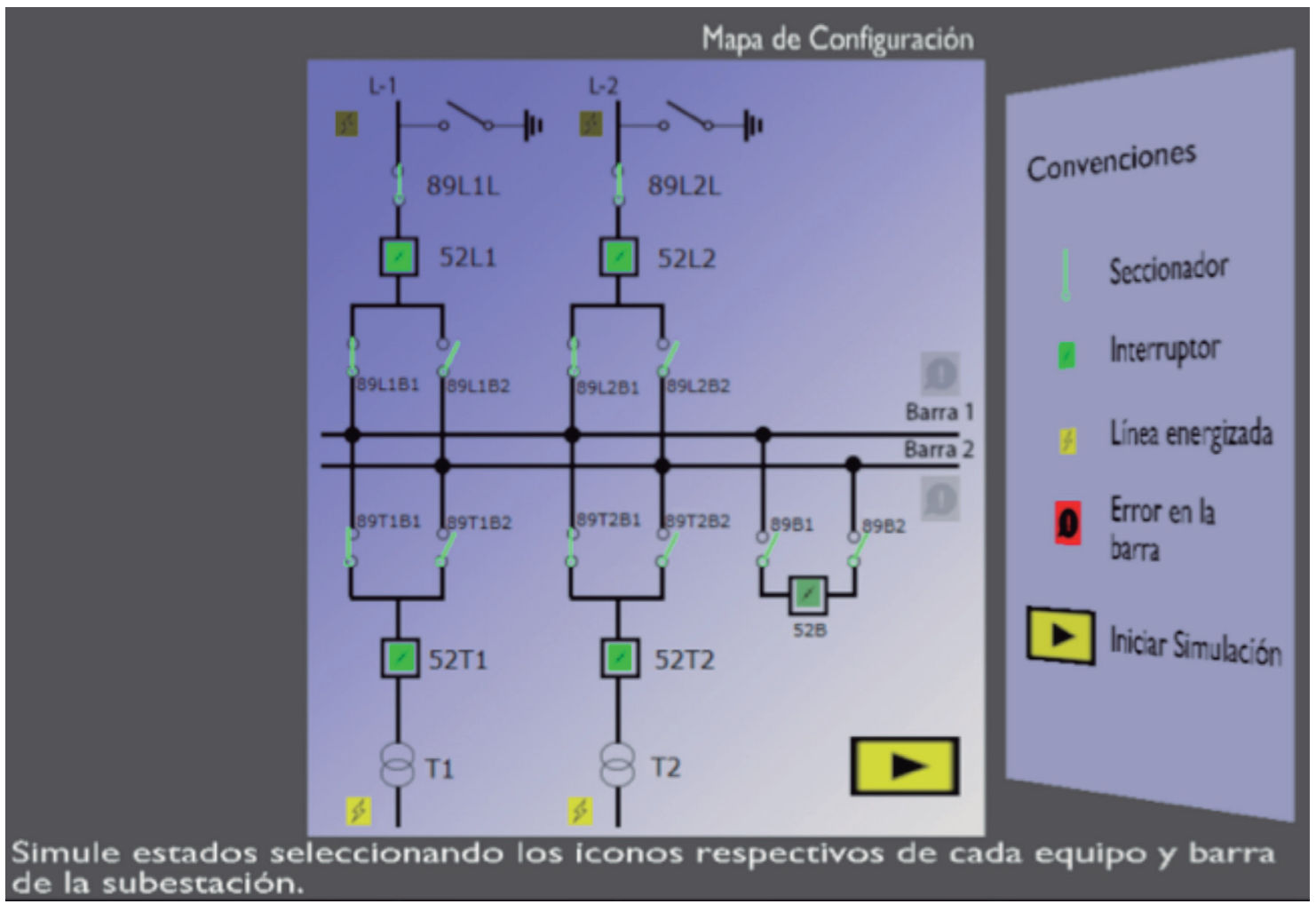

Figura 10. Mapa de configuración de barra doble con cuatro campos Fuente: [5]

Se inicia la simulación y se procede a realizar la maniobra.

La maniobra requerida para realizar mantenimiento en el interruptor 52L1 ES:

- Abrir 51L1.

- Abrir 89L1в1.

- Abrir 89LlL.

Una vez realizada la maniobra en el entorno de simulación, interactúa un listado de las maniobras que se han realizado, junto con la representación visual de la apertura de los seccionadores a las maniobras, tal como lo indica la figura 11.

El estudiante podrá recurrir a esta herramienta virtual como complemento a su formación profesional en el área de subestaciones eléctricas, al experimentar de forma virtual lo que puede ocurrir en un entorno real (como lo son las maniobras eléctricas). En un escenario real es poco viable un autoaprendizaje que permita cometer errores sin las consecuencias que este genera, tales como afectación, tanto de la calidad en el suministro de energía eléctrica, como de la continuidad en la prestación del servicio. Si se analiza desde el punto de vista de la calidad de potencia, podrían aparecer perturbaciones que puedan degradar el funcionamiento de los elementos que conforman el sistema eléctrico de potencia, y en consecuencia, afectar al usuario final. Tales perturbaciones afectan los parámetros de amplitud, frecuencia y forma de onda de tensiones y corrientes presentes en el sistema que, según el tipo, pueden ser largas, lentas o rápidas, y generalmente son variaciones de tensión de larga duración, es decir, subtensiones y sobretensiones que aparecen con cambios de carga y operaciones de maniobra en el sistema. Una sobretensión puede aparecer como resultado de un deslastre de carga por maniobras en la misma, o una mala regulación de la tensión de suministro, mientras que una subtensión se origina al energizar grandes cargas o al desconectar bancos de condensadores. Por tal motivo, no es posible maniobrar este tipo de instalaciones sin un 


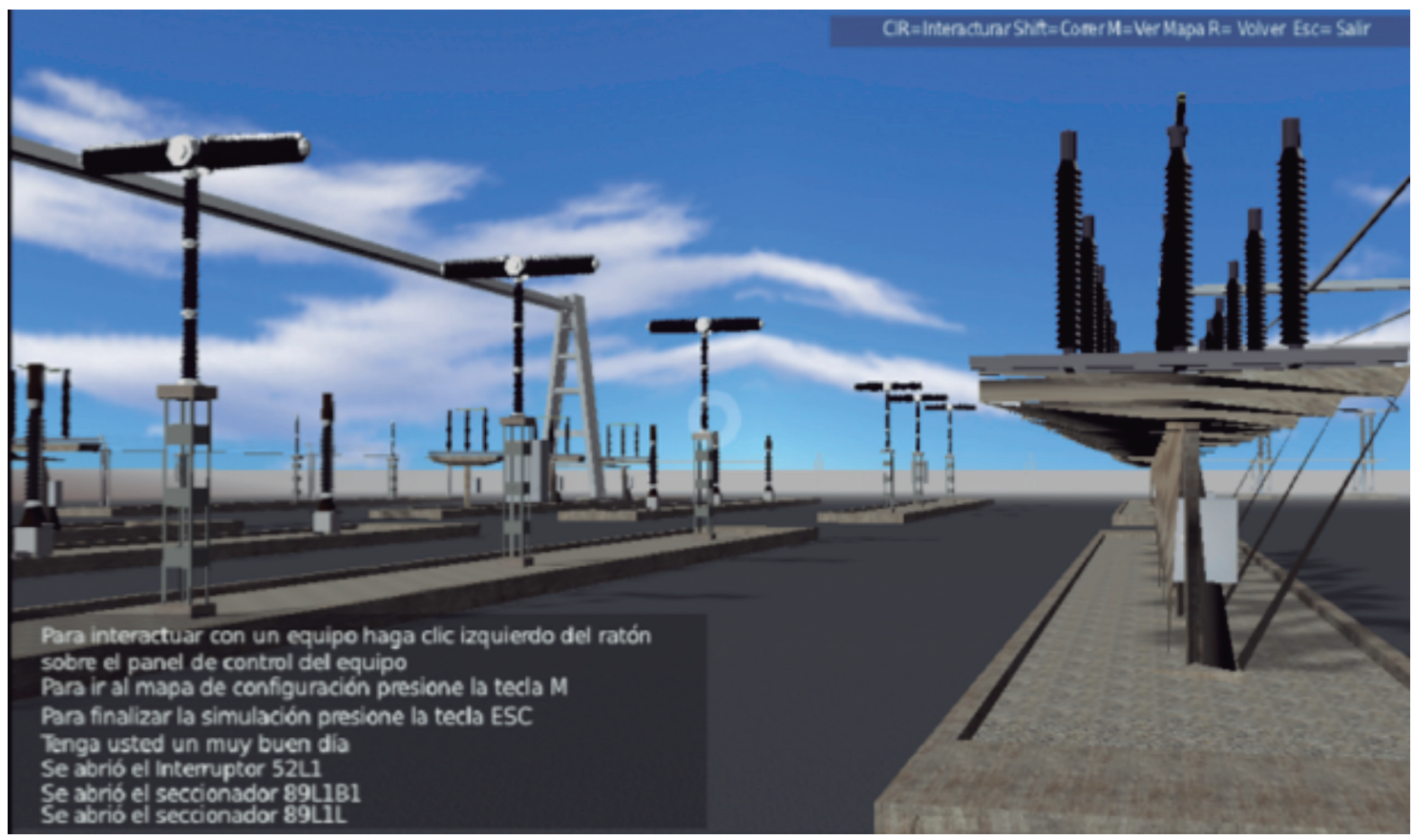

Figura 11. Mapa de configuración de barra doble con cuatro campos Fuente: [5]

entrenamiento previo, el cual garantice que la persona que realizará las maniobras cuente con la experiencia y el conocimiento técnico que evite, en lo posible, accidentes y daños en las instalaciones de la propia subestación, así como de los usuarios finales.

La adopción de un enfoque técnico y pedagógico, como en el caso del simulador, permite un acercamiento a la construcción de herramientas virtuales e incentivar el uso de la metodología virtual mediante laboratorios virtuales para la enseñanza de diferentes temáticas del área de subestaciones eléctricas.

\section{Resultados}

El simulador se empezó a utilizar desde el 2015I, hacia la fecha de elaboración de este artículo (2016-III), por los estudiantes de Subestaciones Eléctricas de la Facultad de Ingeniería del profesor William Alfonso Riaño-Maldonado. Los indicadores de repitencia para el periodo 2015-I al 2016-I muestran una disminución abrupta del 2015-III al 2016-I. Esto debido a que se presentó anormalidad académica para el semestre de 2016-I que, como se explicó anteriormente, flexibiliza la forma de evaluación y minimiza las horas de trabajo autónomo y cooperativo. La tabla 3 expone estas cifras.
Tabla 3. Índices de repitencia de la asignatura subestaciones eléctricas del 2015 al 2016

\begin{tabular}{|c|c|}
\hline Periodo & $\begin{array}{c}\text { Porcentaje de estudiantes que no } \\
\text { aprobaron la asignatura }\end{array}$ \\
\hline $2015-\mathrm{I}$ & $33,3 \%$ \\
\hline $2015-\mathrm{III}$ & $32,5 \%$ \\
\hline $2016-\mathrm{I}$ & $8,7 \%$ \\
\hline
\end{tabular}

Fuente: [28]

Estas cifras no permiten concluir que la implementación del simulador en el aula de clase, con el fin de fortalecer los procesos de enseñanza-aprendizaje, haya contribuido a disminuir los índices de repitencia (tal como se aprecia en la tabla 3), por el factor externo ya mencionado. Sin embargo, la percepción de los estudiantes frente a este desarrollo virtual ha sido bastante positiva, de modo que es posible justificar su validación e implementación.

Una encuesta - cuya ficha técnica se relaciona a continuación en la figura $12-$, realizada a un $27,69 \%$ del total de los estudiantes que cursaron la asignatura de Subestaciones Eléctricas durante el 2016, permite sustentar la validez de la inclusión del simulador en la asignatura como apoyo al proceso de enseñanza-aprendizaje. 


\begin{tabular}{|l|l|}
\hline \multicolumn{2}{|c|}{ Ficha Técnica } \\
\hline Población objetivo & $\begin{array}{l}\text { Estudiantes de Ingeniería Eléctrica que hayan cursado la asignatura de Subestaciones } \\
\text { Eléctricas con el profesor William Riaño de la Facultad de Ingeniería de la Universi- } \\
\text { dad Distrital Francisco José de Caldas. }\end{array}$ \\
\hline Universo representado & $\begin{array}{l}\text { Un total de } 65 \text { estudiantes que cursaron la asignatura de Subestaciones Eléctricas } \\
\text { entre febrero del } 2016 \text { y diciembre del } 2016 .\end{array}$ \\
\hline Técnica & Encuesta mediante formulario de Google. \\
\hline Tamaño de la muestra & $\begin{array}{l}\text { Un total de } 18 \text { estudiantes que cursaban la asignatura, lo que corresponde a un } \\
27,69 \% \text { del total de las personas que la cursaron durante el 2016. }\end{array}$ \\
\hline Momento de aplicación & Del 31 de octubre del 2016 al 15 de noviembre del 2016. \\
\hline
\end{tabular}

Figura 12. Ficha técnica de la encuesta realizada con relación al simulador de subestaciones eléctricas Fuente: elaboración propia

Encuesta sobre la percepción de los estudiantes respecto al simulador de subestaciones eléctricas como metodología virtual de aprendizaje

El objetivo es analizar la percepción que tienen los estudiantes del área de Subestaciones Eléctricas de la Facultad de Ingeniería, de la Universidad Distrital Francisco José de Caldas, con respecto al simulador que lleva el mismo nombre.

1) ¿Considera usted que el simulador de subestaciones eléctricas ayudó a los estudiantes a la correcta ejecución de maniobras eléctricas?

$\bigcirc$ Sí

$\bigcirc$ No

2) ¿Considera usted que el uso del simulador de subestaciones eléctricas mejoró el desempeño de los estudiantes en la asignatura?

Sí

$\bigcirc$ No

3) ¿Considera usted que la temática más difícil de asimilar por parte de los estudiantes son maniobras eléctricas?

$\bigcirc$ Sí

No

4) ¿Cree usted que el simulador de subestaciones eléctricas es una herramienta pedagógica que contribuye al proceso de enseñanza-aprendizaje?

$\bigcirc$ Sí

No

5) ¿Cree usted que los altos índices de repitencia han disminuido debido al uso del simulador como herramienta pedagógica complementaria al trabajo que realiza el docente en el aula de clase? 


\begin{tabular}{|l|}
\hline Sí \\
No \\
\hline 6) Considera que es importante formular otros mecanismos diferentes al uso de simuladores y/o laboratorios virtuales \\
para mitigar la repitencia en el área de subestaciones? \\
\hline Sí \\
No \\
\hline De ser un Sí la respuesta, indique cuáles podrían ser. \\
\hline
\end{tabular}

Figura 13. Encuesta realizada con relación al simulador de subestaciones eléctricas

Fuente: elaboración propia

La figura 14 muestra los resultados de la encuesta anterior. Se puede inferir, de forma general, que más del $70 \%$ de los estudiantes encuestados afirman que el simulador de subestaciones eléctricas fue una herramienta útil para el tema de maniobras eléctricas, el cual presentaba mayor dificultad de aprendizaje entre los estudiantes, y más del $85 \%$ afirmó que es una herramienta que contribuye al proceso de enseñanza-aprendizaje y mejora el desempeño de los estudiantes en la asignatura.

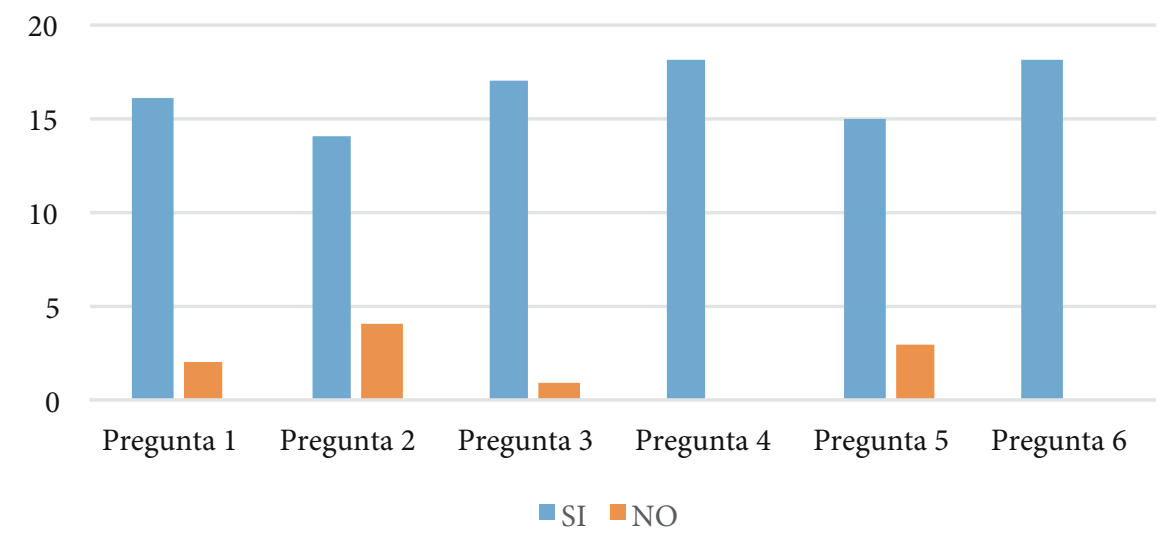

Figura 14. Resultados encuesta sobre el simulador de subestaciones eléctricas Fuente: elaboración propia

Para la pregunta número 6, el $100 \%$ de los estudiantes encuestados consideraron que es importante formular otros mecanismos, diferentes al uso de simuladores, a fin de mitigar la repitencia de estudiantes en su paso por la asignatura. La figura 15 muestra los resultados a la pregunta 6 en caso de ser un Sí la respuesta.

En relación con los resultados de la pregunta $\mathrm{n}^{\circ} 6$ y la gráfica de la figura 15 , un $28 \%$ de los encuestados aseguraron que es necesario un mayor acompañamiento por parte del docente, y lo contemplan como un mecanismo eficaz en el proceso de enseñanza-aprendizaje, y de esta forma mitigar la repitencia. Un $44 \%$ de los encuestados manifestó que es importante tener laboratorios para el área de subestaciones eléctricas, donde se puedan poner en práctica los conocimientos adquiridos en clase y de esta manera evitar repetir la asignatura, ya que se comprenden con más facilidad las temáticas expuestas en la misma. Un $17 \%$ de los 
estudiantes que respondieron la encuesta expresaron que es necesario realizar un trabajo teórico-práctico en armonía con la industria eléctrica, mediante convenios entre academia e industria, para de esta manera lograr una formación que se ajuste a las necesidades de la industria y evitar tener "vacíos" durante el proceso de enseñanza-aprendizaje. Un 11 \% de los encuestados opinaron que sería interesante tener una dinámica en el aula de clase diseñada por el docente que permita a los estudiantes tener gusto por aprender y preguntar, y de esta manera evitar la metodología clásica que se convierte, quizás, en una molestia para los estudiantes, logrando así disminuir los índices de repitencia.

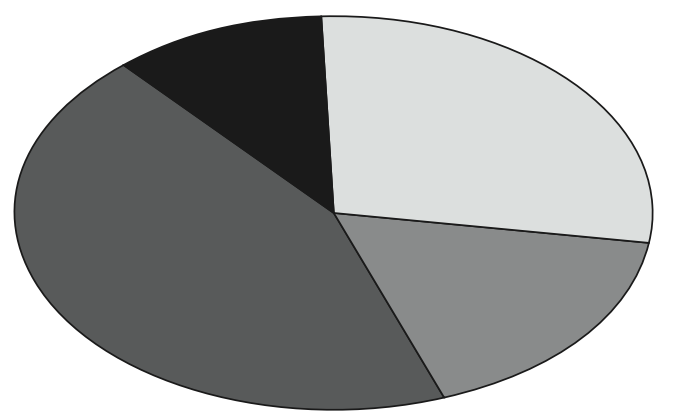

Mayor acompañamiento del docente

Trabajo teórico-práctico con la industria

Adquisición de laboratorios para el área

de subestaciones eléctricas

- Dinámica en el aula de clase

Figura 15. Resultados encuesta sobre el simulador de subestaciones eléctricas: mecanismos para mitigar la repitencia Fuente: elaboración propia

La encuesta realizada a 18 estudiantes que aprobaron la asignatura revela que más de un $40 \%$ de los encuestados proponen adquirir un laboratorio o espacio donde se puedan realizar prácticas relacionadas con las temáticas expuestas en subestaciones eléctricas. Debido a las condiciones de infraestructura de la sede de Ingeniería de la Universidad Distrital Francisco José de Caldas y los altos costos del proyecto, no es posible implementar un laboratorio físico de subestaciones. Pese a ello, con el apoyo de herramientas computacionales se puede lograr un laboratorio virtual, donde el estudiante pueda adquirir habilidades técnicas de modo que, frente a una problemática, identifique causas y efectos $y$, como futuro ingeniero, aporte soluciones que ayuden a mitigar el problema. Los estudiantes están de acuerdo en que el aprendizaje no debe basarse en libros, sino en experiencias, de manera que permitan lograr un mejor desempeño una vez sean profesionales. Es por ello que se sugiere la adopción de un entorno virtual que contenga la mayor cantidad de factores reales simulados, tales como los sonidos producidos en transformadores, líneas, bancos de condensadores, etc., en condiciones normales de operación, falla y mantenimiento, junto con cualquier situación típica real capaz de ser simulada que permita una experiencia enriquecedora a los estudiantes.

El simulador incentiva la creación de nuevos entornos de simulación en ingeniería eléctrica a partir de software libre, de acercar a los estudiantes a los elementos presentes en una subestación eléctrica, así como de su distribución en la misma y, finalmente, resolver dudas y afianzar conocimientos por medio de este simulador. Este artículo es el resultado de un análisis sobre el aporte de la inclusión del simulador, presentado como laboratorio virtual para el área de subestaciones eléctricas en la formación de ingenieros electricistas.

La investigación ahonda no solo en demostrar que el simulador de subestaciones eléctricas es una herramienta virtual pedagógica válida, la cual puede ayudar a reducir los índices de repitencia en la asignatura, sino también en analizar la posibilidad de convertir este simulador en un objeto virtual de aprendizaje (ova). Según el Ministerio de Educación Nacional y algunas instituciones de educación superior, el concepto se define como "un conjunto de recursos digitales, autocontenible y reutilizable, con un propósito educativo y constituido por al menos tres componentes internos: contenidos, actividades de aprendizaje y elementos de contextualización. El objeto de aprendizaje debe tener una estructura de información externa (metadatos) que facilite su almacenamiento, identificación y recuperación" [30]. Por tanto, es necesario tener como mínimo los tres elementos mencionados en el que expresen de manera clara y concreta lo que el estudiante va a aprender. Para el tema de maniobras eléctricas y en general para el diseño de subestaciones, es necesario contar con múltiples formas de mostrar conocimiento al estudiante.

El siguiente análisis muestra algunas de las temáticas de la asignatura en la que es posible implementar entornos virtuales de aprendizaje y, de esta manera, mejorar la última versión del simulador. 
Configuraciones. Elaborar animaciones para cada una de las distintas configuraciones de subestaciones eléctricas con enlaces a fuentes externas, lo cual promueva la lectura de textos científicos sobre nuevas instalaciones de alta tensión, con el objetivo de que el estudiante comprenda su funcionamiento.

Maniobras eléctricas. Tal como se presenta en el simulador, la integración de modelos, animaciones e interfaces hace posible ejecutar maniobras eléctricas bajos los lineamientos básicos para la apertura y cierre de seccionadores e interruptores. Sin embargo, es necesario ampliar las herramientas a las demás configuraciones existentes en instalaciones eléctricas de alta tensión, con el objetivo de que el estudiante logre ejecutar tales maniobras en cualquier tipo de configuración y en situaciones que varíen el clima.

Sobretensiones. Es necesario elaborar animaciones respecto a los diferentes tipos de impulsos (maniobra, descarga atmosférica o temporal), y presentar modelos interactivos en $3 \mathrm{D}$ de los descargadores de sobretensión, a fin de que el estudiante pueda comprender los principios básicos de funcionamiento de los elementos que interactúan, por ejemplo, cuando un impulso de tensión supera la tensión máxima admisible en un equipo.

Niveles de aislamiento. Es importante diseñar un módulo que presente al estudiante los conceptos relacionados con los niveles de protección, una vez este último comprenda el funcionamiento de los descargadores de sobretensión y la incidencia de los diferentes tipos de impulsos en los elementos que componen una subestación eléctrica.

Coordinación de aislamiento. Este módulo se centra más en el diseño, por lo que una vez comprendidas las temáticas anteriormente mencionadas, el estudiante ya es capaz de seleccionar el descargador, estimar un nivel de protección y calcular los niveles básicos de aislamiento para impulso tipo rayo y tipo maniobra. Para este módulo se propone mostrar al estudiante, paso a paso, los cálculos de los niveles de protección y determinar si este ha hecho o no una buena coordinación de aislamiento.

Distancias de seguridad y distancias minimas. Con el fin de garantizar la seguridad de los equipos y de las personas que laboran en las subestaciones, se propone crear avatares que permitan al estudiante conocer las distancias de seguridad para partes y si son rígidas o flexibles.
Dimensionamiento de conductores, barras $y$ cables de guarda. En este módulo se propone la inclusión de catálogos de fabricantes y modelos animados de conductores, barras y cables de guarda, lo cual permita al estudiante dimensionarlos mediante cálculos de potencias y corrientes para una adecuada elección de estos elementos, en concordancia con los parámetros establecidos anteriormente.

Sistemas depuesta a tierra. Se proponela creación de modelos en 3D para el diseño de mallas cuadradas o rectangulares, con o sin electrodos, que permitan al estudiante evaluar los potenciales que puedan aparecer en el terreno y el riesgo que pueda presentar la instalación de puesta a tierra, de tal manera que se verifique el cumplimiento de todos los requerimientos técnicos y de seguridad, según sea el caso.

Métodos de apantallamiento. Al igual que los demás módulos, se proponen escenarios virtuales que proyecten las zonas protegidas con elementos tales como cables de guarda, puntas franklin o mástiles, utilizando el método electro geométrico o el método empírico de ángulos fijos, todo basado en modelación de ingeniería tridimensional.

Las anteriores son algunas sugerencias que podrían enfocar el simulador de subestaciones eléctricas al concepto de objeto virtual de aprendizaje (ova), lo que potencializaría la utilidad de la herramienta como parte del proceso de enseñanza-aprendizaje.

\section{Discusión}

La construcción de software para fines educativos ha permitido, en los últimos años, fortalecer los procesos de enseñanza-aprendizaje en distintas instituciones de educación superior. Los resultados del presente estudio revelan que el uso del simulador generó una percepción positiva en los estudiantes de subestaciones eléctricas. Según los resultados de la encuesta realizada, quizás contribuyó a la disminución de los índices de repitencia, debido a que el simulador se enfocó en la didáctica para la ejecución de maniobras eléctricas, aclarando que la principal causa de disminución de estas cifras se debió a la anormalidad académica, evento que transcurrió en el primer semestre del 2016. El simulador apunta a solucionar la problemática que se manifestó como la dificultad en aprender la correcta ejecución de 
maniobras en una subestación, de acuerdo con su configuración, las cuales se basan en la apertura y cierre de interruptores y seccionadores.

Si bien algunos pueden afirmar que un simulador nunca será igual a realizar la labor en campo, puesto que existen condiciones que hasta la fecha son complejas de simular (la sensación de ionización del aire, la presión por la responsabilidad que implicaría dejar sin servicio una parte del sistema eléctrico e interacciones con jefes o técnicos a cargo, entre otras), se debe considerar el simulador como una herramienta pedagógica que busca ayudar al estudiante con el estudio de maniobras, mas no es una sustitución de una situación laboral.

El propósito del artículo es mostrar la potencialidad del simulador como laboratorio virtual para fortalecer los procesos de enseñanza-aprendizaje en el área de subestaciones eléctricas, así como dar un soporte válido sobre las ventajas que se obtuvieron al implementarlo en el aula de clase. Su investigación no se extiende a analizar la incidencia de otros factores en los índices de repitencia y se deja abierta para futuros análisis.

Para el continuo desarrollo del simulador, se sugiere que se enfoque en tres aspectos: el primero, orientado a la adquisición de conocimiento asertivo mediante módulos de evaluación que permitan conocer con certeza el aprendizaje del estudiante; el segundo, a la participación de todos los usuarios mediante un enfoque colaborativo en el que se evidencie el trabajo en equipo; y un tercero con la "creación de conocimiento", en el cual se desarrollen e intercambien prácticas [10] en relación con el mantenimiento de subestaciones eléctricas (maniobras eléctricas). La ampliación del mismo sugiere una biblioteca de imágenes reales sobre los diferentes elementos que conforman una subestación, tales como interruptores de potencia (figura 16), transformadores de instrumentos y seccionadores (figura 17).

De la misma forma, se sugiere un módulo que permita ver las señales de tensión y corriente después de realizar alguna operación que pueda afectar la calidad de potencia en la subestación eléctrica, tal como se muestra para una instalación real (figura 18).

Se propone la inclusión de todas las configuraciones en el simulador de subestaciones eléctricas en tendencia europea (barra sencilla, barra principal y transferencia, doble barra, doble barra más seccionador de "by-pass", doble barra más seccionador de transferencia, doble barra más barra de trasferencia y malla), y en tendencia americana (anillo, interruptor y medio, y doble interruptor), como una propuesta de desarrollo virtual y educativo con énfasis en ingeniería eléctrica, según se muestra en las figuras 19, 20 y 21.

Asimismo, sería importante incluir la parte de diseño con un enfoque técnico-pedagógico, ya que en la actualidad existe diversidad de software que permite realizarlo, pero su alcance es limitado para estudiantes por razones de costos. Este diseño incluiría módulos para sistema de puesta a tierra (determinar tensiones de paso y contacto bajo condiciones de falla, valores de resistividad y resistencia del cuerpo humano, selección de electrodos de acuerdo con las características del suelo, materiales de los mismos, geometría del terreno y condiciones ambientales (figura 22), coordinación de aislamiento (presentación de los diferentes tipos de aisladores, fabricantes, características eléctricas y mecánicas, estimación del BsL y el BIL, (figura 23), y métodos de apantallamiento (presentación de elementos de protección tales como cables de guarda, puntas de Franklin, mástiles y diferentes métodos como el método empírico de los ángulos fijos, o el método electrogeométrico, de tal manera quela efectividad de cualquiera de los métodos pueda ser vista por el estudiante en visualización 3D, (figura 24). Una vez más se mediría el impacto en los estudiantes, con una versión mejorada del simulador que incluya los módulos expuestos, mediante resultados de los exámenes aplicados para los diferentes temas de la asignatura, indicadores de repitencia y evaluación de la percepción de los estudiantes frente al uso de herramientas virtuales, con el fin de fortalecer su aprendizaje mediante mecanismos alternativos para la comprensión de los diferentes temas expuestos. El objetivo de incluir módulos como los mencionados anteriormente es que el simulador también tenga apoyo en las temáticas de diseño, y no se centre solo en operación y mantenimiento de subestaciones eléctricas. También está abierta la discusión para proponer otras metodologías que suplan las necesidades de experimentar y que, en conjunto con herramientas tecnológicas, puedan cumplir el requerimiento técnico que exige la asignatura, en el propósito de lograr una formación profesional íntegra que consolide los conocimientos teóricos mediante la experimentación. 


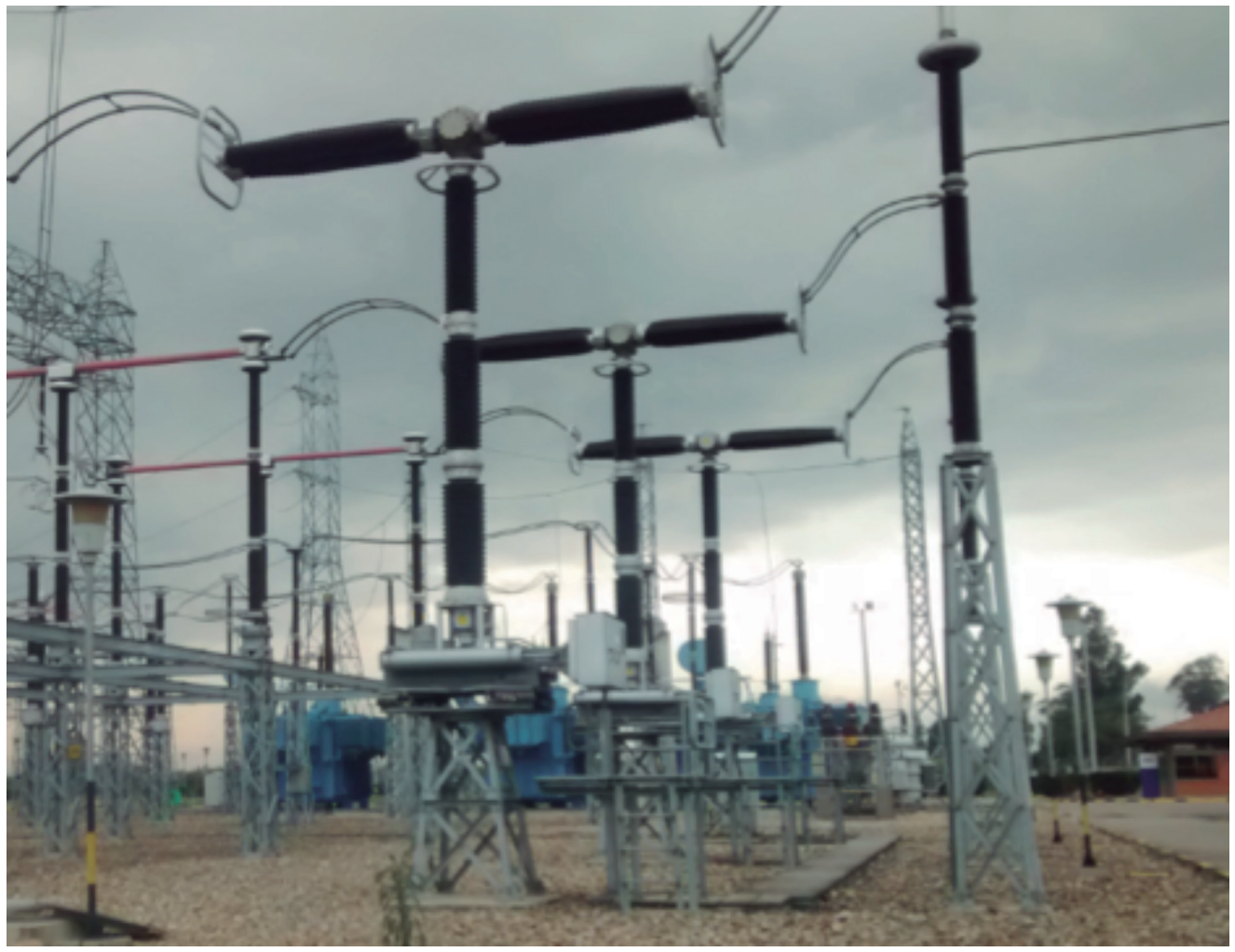

Figura 16. Interruptor de potencia. Subestación Bacatá

Fuente: fotografía de Diego Hernando Nausan-García, tomada del 23 de octubre del 2015
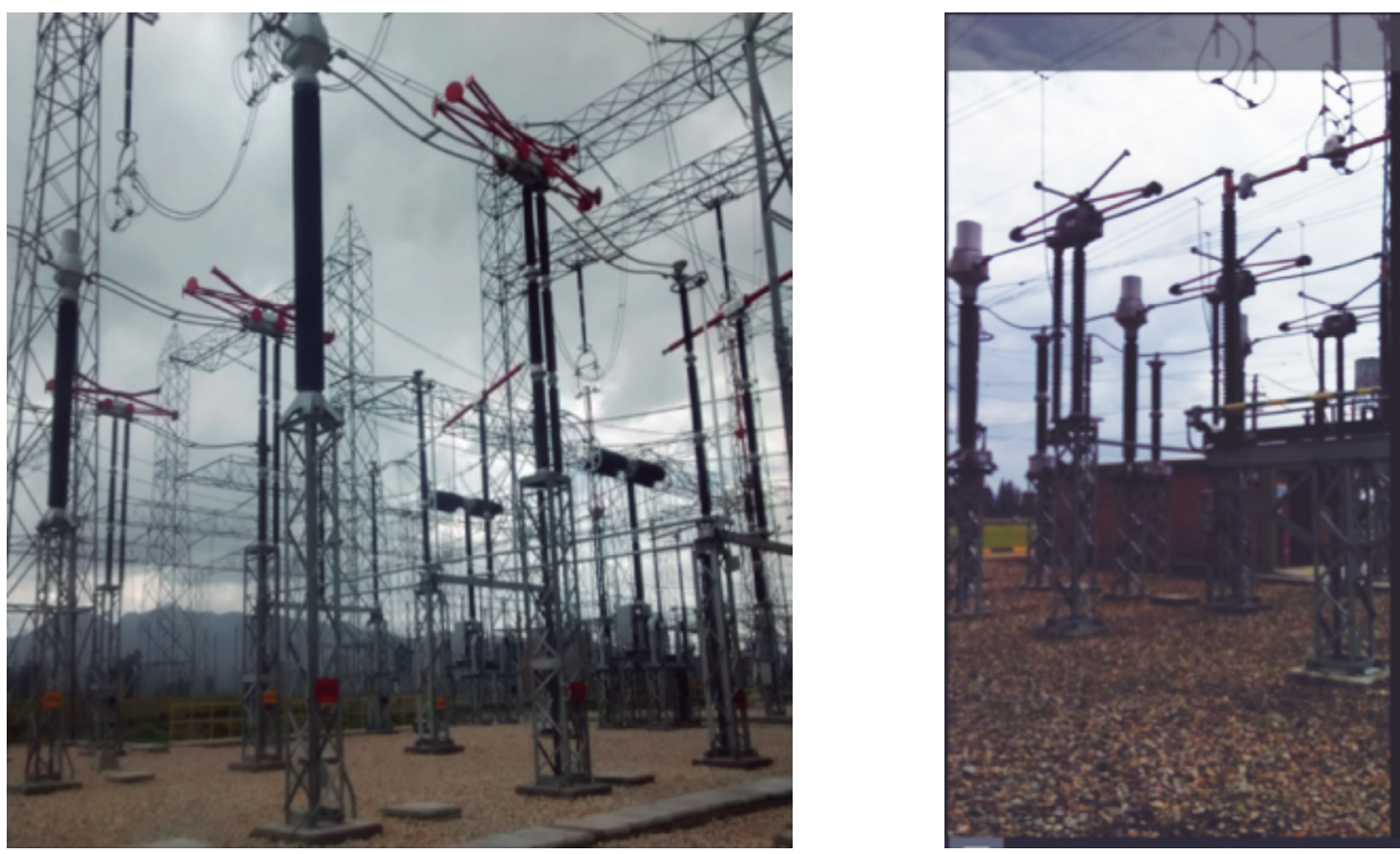

Figura 17. Transformador de instrumento y seccionador tipo pantógrafo. Subestación Bacatá Fuente: fotografía de Diego Hernando Nausan-García, tomada el 23 de octubre del 2015 


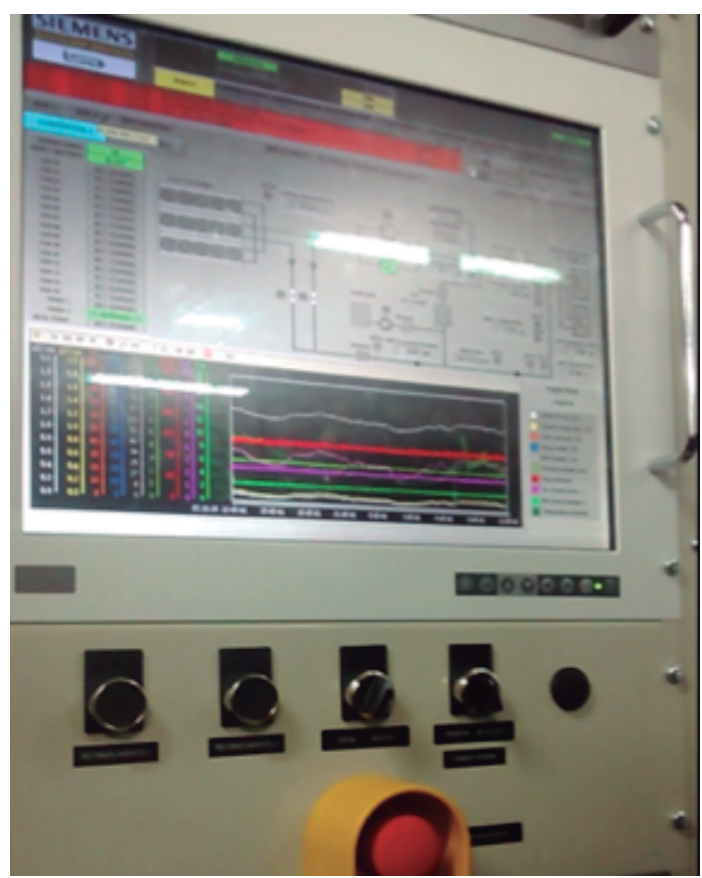

Figura 18. Mímico de la subestación eléctrica Bacatá

Fuente: fotografía de Diego Hernando Nausan-García, tomada el 23 de octubre del 2015

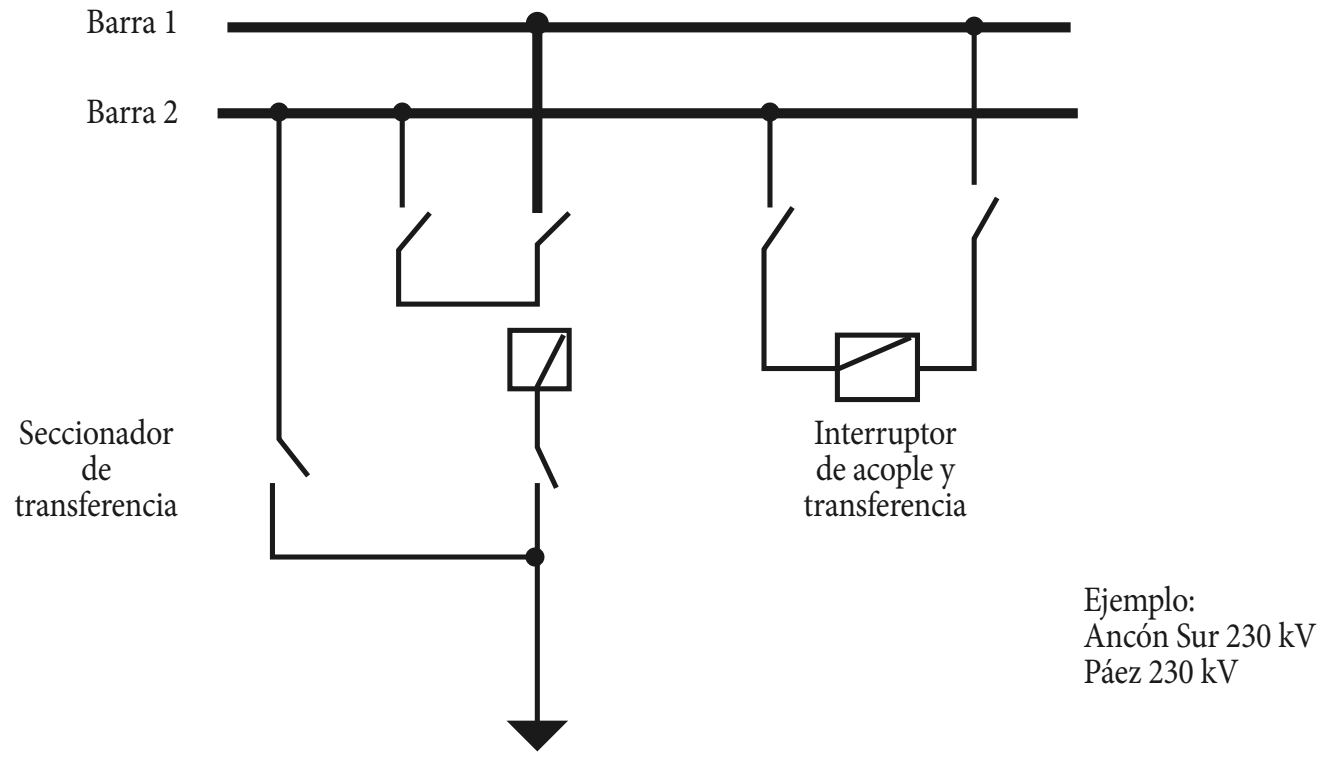

Figura 19. Configuración de barra doble + seccionador de transferencia Fuente: [31] 


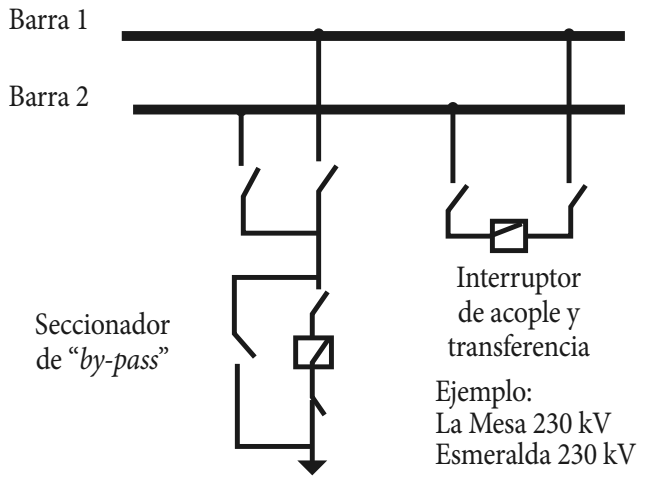

Configuración de barra doble con by-pass

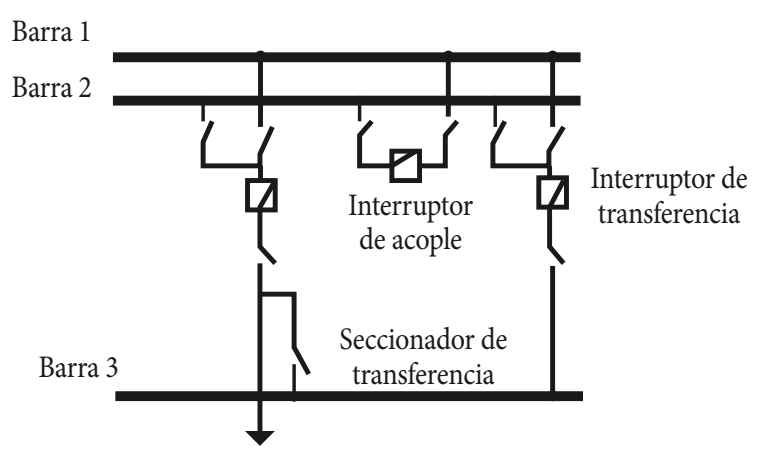

Configuración de barra doble + Barra de transferencia

Figura 20. Configuración de barra doble con by-pass y barra doble + barra de transferencia Fuente: [31]

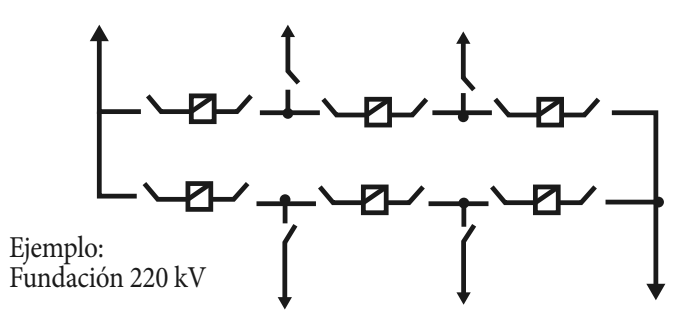

Configuración en anillo

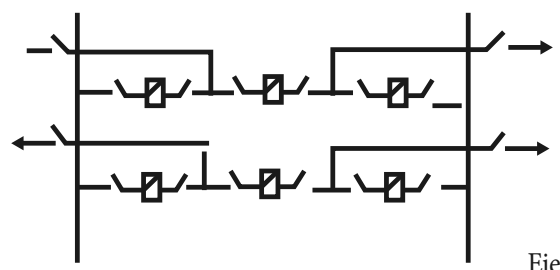

Barra 1
Ejemplo:

San Carlos $230 \mathrm{~W}$

Barra 2 Primavera $230 \mathrm{~W}$

Configuración en interruptor y medio

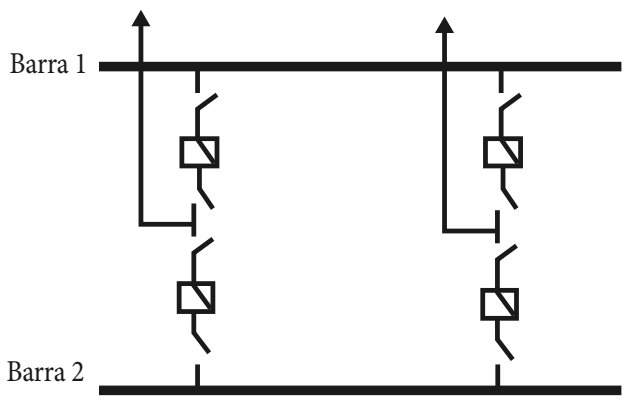

Configuración en doble interruptor

Figura 21. Configuración en anillo, interruptor y medio y doble interruptor Fuente: [31] 

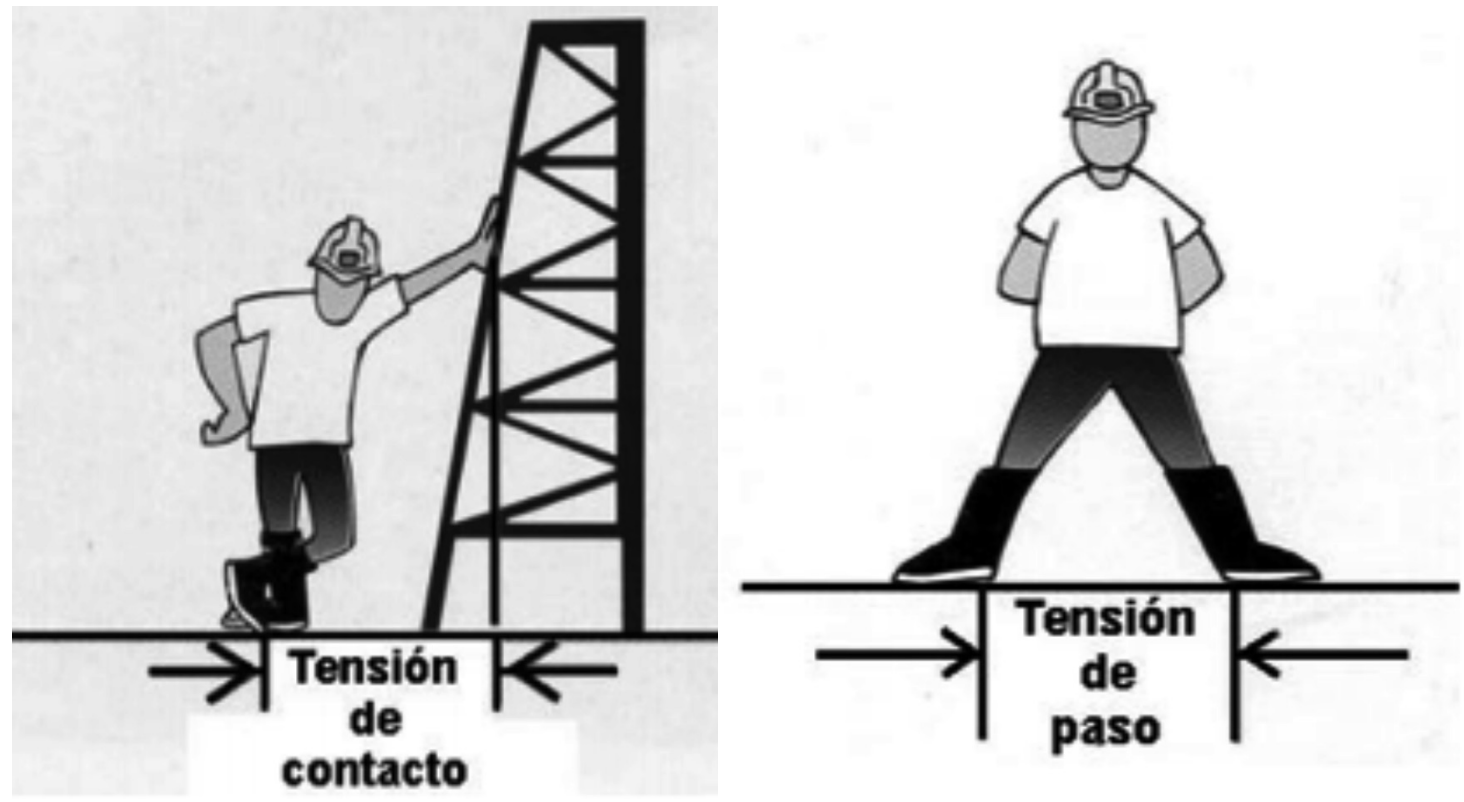

Figura 22. Módulos para sistemas de puesta a tierra Fuente: [32]

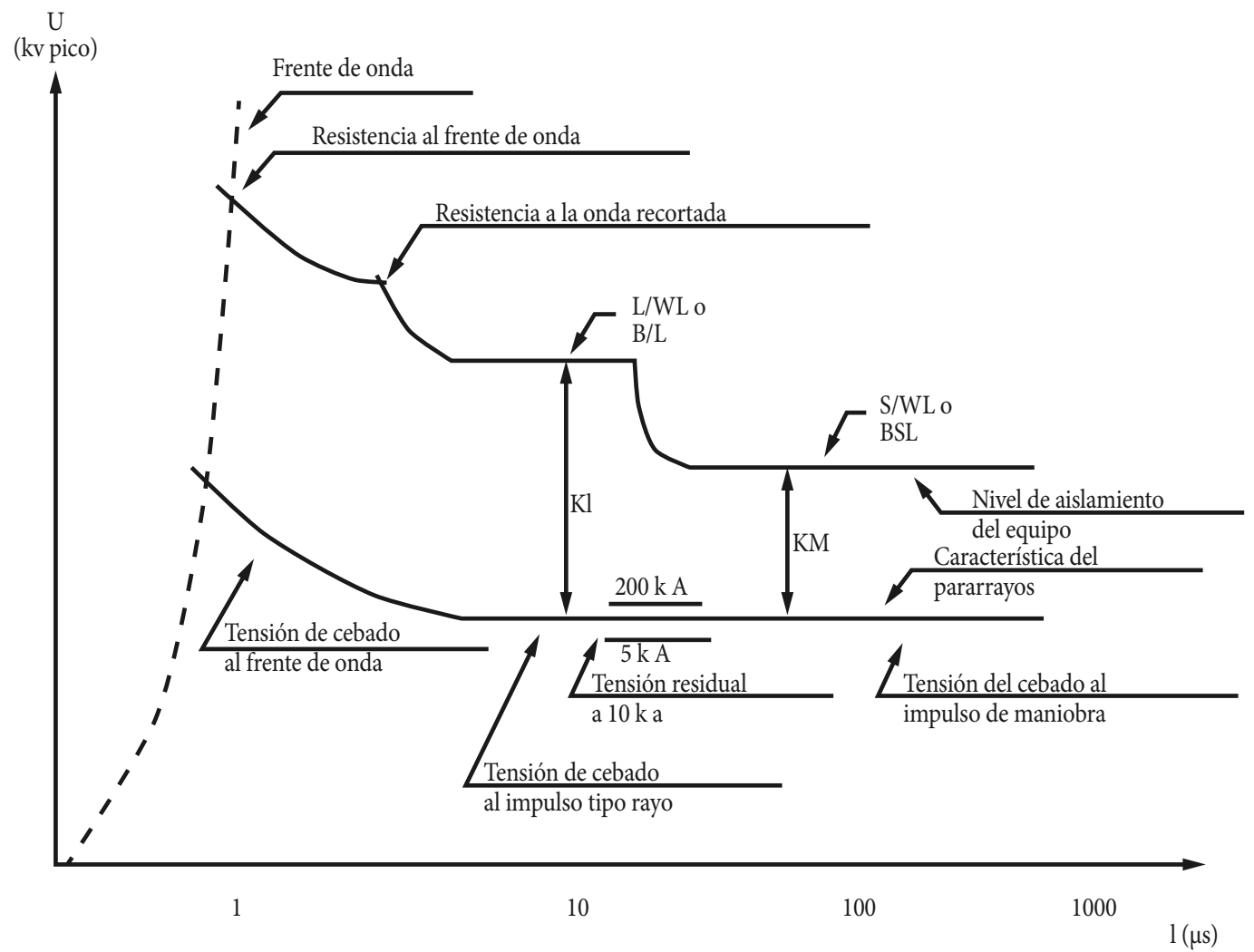

Figura 23. Módulos para coordinación de aislamiento Fuente: [33] 


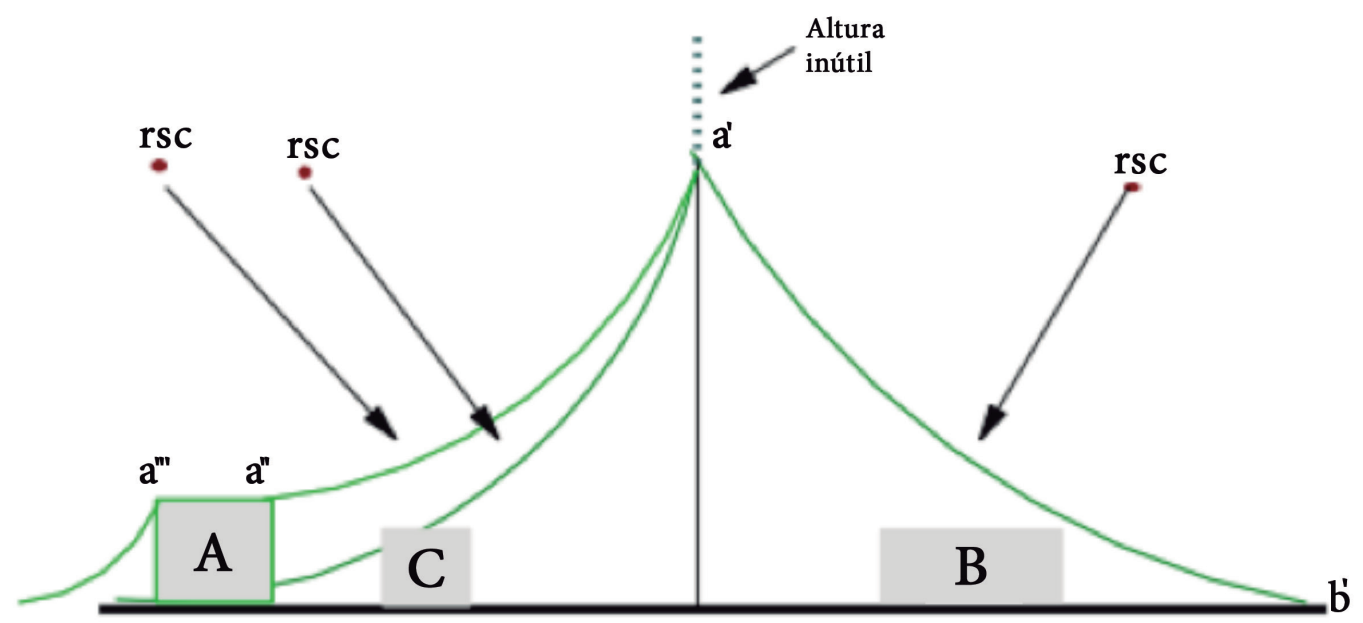

Figura 24. Módulos para métodos de apantallamiento Fuente: [32]

\section{Conclusiones}

El apoyo pedagógico de los laboratorios virtuales en el proceso de enseñanza-aprendizaje para la comprensión y experimentación de los temas vistos en una asignatura, ha sido una forma eficaz de aprendizaje, especialmente, en áreas en las que la experimentación es un elemento clave en la formación de un buen profesional, como en el caso de subestaciones eléctricas para la formación de ingenieros electricistas. El simulador desarrollado en la Facultad de Ingeniería de la Universidad Distrital Francisco José de Caldas, en colaboración con estudiantes de Ingeniería de Sistemas, permitió demostrar la utilidad de esta herramienta virtual para la comprensión de los procesos de operación y mantenimiento de instalaciones de alta y extra alta tensión (subestaciones eléctricas), además de contribuir a la reducción de los índices de repitencia de la asignatura. Con base en los resultados del presente estudio, se puede concluir que los laboratorios virtuales han sido y serán una herramienta complementaria válida en la formación de estudiantes de ciencias e ingenierías, al facilitar la comprensión de las temáticas y mejorar su desempeño en diferentes asignaturas.

El simulador de subestaciones eléctricas permitió a los estudiantes conocer elementos propios de una instalación eléctrica en alta tensión, tales como transformadores de instrumentos, transformadores de potencia, seccionadores, interruptores de potencia que por su limitado acceso es difícil conocerlos físicamente, la comprensión de las principales tendencias de configuración (barra sencilla y doble barra), así como experimentar (ejecutando maniobras eléctricas) las veces que consideraran necesarias, sin riesgos de accidente. Esto en consideración a la guía del docente en un trabajo conjunto con el uso de herramientas pedagógicas virtuales, de modo que facilite el proceso de enseñanza-aprendizaje. La visualización en 3D y la descripción de los diferentes elementos ayudó en la comprensión del dimensionamiento de una instalación eléctrica que maneja alta y extra alta tensión. El apoyo colaborativo entre estudiantes de la asignatura, la consulta de textos didácticos, o incluso visitas en diferentes sitios web con videos o conferencias como métodos de aprendizaje, han sido elementos complementarios al simulador de subestaciones como laboratorio virtual que le ha permitido a los estudiantes interactuar y experimentar en un ambiente virtual que asemeja la realidad.

\section{Referencias}

[1] D. Chorafas \& H. Steinmann, Realidad virtual; aplicaciones prácticas en los negocios y en la industria, 1a edición, Ciudad de México DF, Prentice Hall \& IBD, pp. 300-317, 1996. 
[2] L. A. Luengas, J. C. Guevara \& G. Sánchez, “¿Cómo desarrollar un laboratorio virtual? Metodología de diseño", Nuevas ideas en Informática Educativa, vol. 5, pp. 165-170, 2009. Disponible en: http://www.tise. cl/volumen5/TISE2009/Documento20.pdf

[3] C. Infante Jiménez, "Propuesta pedagógica para el uso de laboratorios virtuales como actividad complementaria en las asignaturas teórico-prácticas," Rev. Mex. Investig. Educ., vol. 19, pp. 917937, 2014. Disponible en: http://www.redalyc.org/ pdf/140/14031461013.pdf

[4] J. M. Andujar \& T. J. Mateo, "Diseño de Laboratorios Virtuales y/o Remotos. Un caso práctico," Rev. Iberoam. Automática e informática Ind., vol. 7, pp. 6472, 2010. Disponible en: http://www.sciencedirect. com/science/article/pii/S1697791210700091.

[5] J. J. Carvajal Villalobos \& W. F. Ardila Guzmán, Desarrollo de entornos virtuales interactivos de configuraciones de subestaciones eléctricas de barra simple y doble barra, como material de apoyo educativo, Universidad Distrital Francisco José de Caldas, 2015.

[6] A. Medina \& G. Saba, "Los laboratorios virtuales y los laboratorios remotos en la enseñanza de la ingeniería”, Rev. Educación en Ing., vol. 4, pp. 24-31, 2011. Disponible en: http://bibliografia.eovirtual. com/LorandiA_2011_Laboratorios.pdf.

[7] L. O. Flowers, "Investigating the Effectiveness of Virtual Laboratories in an Undergraduate Biology Course", J. Hum. Resour. Adult Learn., vol. 7, diciembre, pp. 110-116, 2011. Disponible en: http:// connection.ebscohost.com/c/articles/78856956/investigating-effectiveness-virtual-laboratories-undergraduate-biology-course

[8] M. W. Dunham, K. Ghirtis \& M. Beleh, “The use of virtual laboratories and other web-based tools in a drug assay course," Am. J. Pharm. Educ., vol. 76, n. ${ }^{\circ}$ 5, 2012. doi: 10.5688/ajpe76584.

[9] S. Amirkhani, "Design and Implementation of Interactive Virtual Control Laboratory Using Haptic Interface for Undergraduate Engineering Students", Wiley Period. Inc, pp. 508-518, 2016. doi: 10.1002/ cae. 21727

[10] R. Molina-Vásquez, Construcción del concepto de tecnología en una red virtual de aprendizaje, Universidad Distrital Francisco José de Caldas, 2014.

[11] A. P. Lorandi-Medina, A. C. García-Reynoso, G. Hermida-Saba \& E. Ladrón de Guevara-Durán, “Canihuá: Un portal de Laboratorios Virtuales", Inst. Tecnológico Orizaba, pp. 1-6, 2011. Disponible en: http://www.uv.mx/personal/alorandi/files/ 2011/11/ Canihu\%C3\%A1-Un-portal-de-Laboratorios-Virtuales.pdf.
[12] E. E. Toth, "Analyzing 'real-world' anomalous data after experimentation with a virtual laboratory", Educ. Technol. Res. Dev., vol. 64, n.o 1, pp. 157-173, 2016. Disponible en: http://link.springer.com/article/10.1007/s11423-015-9408-3.

[13] A. Alexiou, C. Bouras, \& E. Giannaka, "Virtual Laboratories in Education: A cheap way for schools to obtain laboratories for all courses by using the Computer Laboratory," en Technology Enhanced Learning IFIO International Federation for Information Processing (IFIPAICT), J. P. Courtiatry, C. Davarakis \& T. Villemur Eds., Tolosa, Francia, pp. 19-28, 2005. doi: https://doi.org/10.1007/0-387-24047-0_2

[14] J. M. Harley, E. G. Poitras, A. Jarrell, M. C. Duffy \& S. P. Lajoie, "Comparing virtual and location-based augmented reality mobile learning: emotions and learning outcomes," Educ. Technol. Res. Dev., vol. 64, n.o 3, pp. 359-388, 2016. Disponible en: http://link. springer.com/article/10.1007/s11423-015-9420-7

[15] (ICL) International Conference on Interactive Collaborative Learning, "The Application of Virtual Reality Technologies in Engineering Education for the Automotive Industry", IEEE Inst. Electr. Electron. Eng, septiembre, pp. 536-544, 2015. doi: https://doi. org/10.1109/ICL.2015.7318086.

[16] V. Potkonjak, M. Gardner, V. Callaghan, P. Mattila, K. Jovanovi, C. Guetl et al., "Virtual laboratories for education in science, technology, and engineering: A review", Comput. Educ., vol. 95, pp. 309-327, 2016. Disponible en: http:// www.sciencedirect.com/science/article/pii/S0360131516300227

[17] C. A. Jara, F. A. Candelas \& F. Torres, "Virtual and Remote Laboratory for Robotics ELearning," Comput. Aided Chem. Eng., vol. 25, pp. 1193-1198, 2008. doi: https://doi.org/10.1016/S1570-7946(08)80205-2

[18] A. N. Nithyaa, R. Premkumar, S. Dhivya \& M. Vennila, "A Real Time Foot Pressure Measurement for Early Detection of Ulcer Formation in Diabetics Patients Using Labview", Procedia Eng., vol. 64, pp. 13021309, 2013. Disponible en: http://www.sciencedirect. com/science/article/pii/S1877705813017256.

[19] M. Kliment, R. Popovic \& J. Janek, "Analysis of the Production Process in the Selected Company and Proposal a Possible Model Optimization Through PLM Software Module Tecnomatix Plant Simulation", Procedia Eng, vol. 96, pp. 221-226, 2014. doi: https:// doi.org/10.1016/j.proeng.2014.12.147

[20] P. TrebuEa, R. Popovib \& S. Káos, "Methodology of the Creation of Human and Robot Operation in the Tecnomatix Process Simulate", Procedia Eng., vol. 96, pp. 483-488, 2014. doi: https://doi.org/10.1016/j. proeng.2014.12.119 
[21] E. Vega, G. Muñoz, H. Roncancio, H. Velasco, J. Ballen \& C. Rodríguez, "Laboratorio remoto en la Educación de la Ingeniería," Acad. y Desarro., vol. 7, n.o 1, pp. 85-87. Disponible en: http://revistas.udistrital.edu.co/ ojs/index.php/reving/ article/view/2276/3072.

[22] S. Xudong, J. Tao \& W. Liwen, "Research on Aircraft Cable Defects Locating Method Based on Time-Frecuency Domain Reflection", Procedia Eng., vol. 17, pp. 446-454, 2011. doi: https://doi.org/10.1016/j. proeng.2011.10.054

[23] E. Upton \& C. Severance, "Eben Upton: Raspberry Pi," IEEE Inst. Electr. Electron. Eng., pp. 1-5, 2013. doi: https://doi.org/10.1109/MC.2013.349.

[24] J. C. Castañeda-Alvarado \& G. J. R. Piñeros-Mora, Diseño y análisis de conectividad de una plataforma educativa virtual piloto en 3 D para un laboratorio de simulación de nanotecnología sobre la red Rita-UD mediante software libre y QOS, Universidad Distrital Francisco José de Caldas, 2012.

[25] A. Radionov, A. Podoltsev \& A. Zahorulko, "Finite-Element Analysis of Magnetic Field and the Flow of Magnetic Fluid in the Core of Magnetic-Fluid Seal for Rotational Shaft", Procedia Eng., vol. 39, pp. 327338, 2012. Disponible en: http://www.sciencedirect. com/science/article/pii/S1877705812024241
[26] B. A. Rachedi, A. Babouri \& F. Berrouk, "A study of electromagnetic field generated by high voltaje lines using COMSOL MULTiPHysics", IEEE Inst. Electr. Electron. Eng., pp. 1-5, 2013. doi: https://doi.org/10.1109/ CISTEM.2014.7076989.

[27] O. K. P. Mokoka \& K. O. Awodele, "Reliability Evaluation of Distribution Networks Using NEPLAN \& DIgSILENT Power Factory”, IEEE Inst. Electr. Electron. Eng., pp. 1-5, 2013. doi: https://doi.org/10.1109/ AFRCON.2013.6757621

[28] Oficina Asesora de Sistemas (OAs) Universidad Distrital Francisco José de Caldas, Índices de repitencia subestaciones eléctricas, Bogotá D.C., 2016.

[29] SafeCreative, "Navegapolis", 2015, 2015. Disponible en: http://navegapolis.com

[30] Ministerio de Educación Nacional, Objetos virtuales de aprendizaje e Informativos 2015, 2015.

[31] xм, Expertos en Mercados, Configuración de Subestaciones Eléctricas, 2015.

[32] J. Romero \& F. Vega, "Protecciones eléctricas, apuntes de clase," $11^{a}$ edición, Bogotá, Editorial Universidad Nacional de Colombia, 2000, pp. 224-225.

[33] Protección contra rayos, principios generales, NTC 4552, 2004. 